\title{
A Dynamic Mean-Variance Analysis for Log Returns
}

\author{
Min Dai \\ Department of Mathematics, Risk Management Institute, and Suzhou Research Institute, National University of Singapore, 10 \\ Lower Kent Ridge Road, Singapore 119076. matdm@nus.edu.sg \\ Hanqing Jin \\ Oxford-Nie Financial Big Data Laboratory, Mathematical Institute, The University of Oxford, Woodstock Road, Oxford, OX2 \\ 6GG, U.K. jinh@maths.ox.ac.uk \\ Steven Kou \\ Department of Finance, Questrom School of Business, Boston University, 595 Commonwealth Avenue Boston, MA 02215. \\ kou@bu.edu \\ Yuhong Xu \\ Center for Financial Engineering, Math Center for Interdiscipline Research, and School of Mathematical Sciences, Soochow \\ University, Suzhou 215006, P. R. China. yuhong.xu@hotmail.com
}

\begin{abstract}
We propose a dynamic portfolio choice model with the mean-variance criterion for log-returns. The model yields time-consistent portfolio policies and is analytically tractable even under some incomplete market settings. The portfolio policies conform with conventional investment wisdom (e.g. richer people should invest more absolute amount of money in risky assets; the longer investment time horizon, the more proportional amount of money should be invested in risky assets; and for long-term investment, people should not short sell major stock indices whose returns are higher than the risk-free rate), and the model provides a direct link with the CRRA utility maximization in a complete market.
\end{abstract}

Key words: portfolio choices, stochastic volatility, time-varying mean returns, risk aversion recovery

History: First version: Apr 2017. This version: Aug 2019

\section{Introduction}

Portfolio optimization has a long history in finance, dating back to celebrated works of single period mean-variance analysis by Markowitz [47] and continuous-time expected utility maximization by Merton [49. However, both the expected utility maximization model and the mean-variance model need further improvements.

For example, in a utility maximization model, it is difficult to estimate investors' risk profile, which is given by the utility function 1 On the other hand, although one can easily elicit investors' mean variance parameters in the single period model by asking the investor

\footnotetext{
${ }^{1}$ Even for a special case like CRRA utility function, the estimation of the risk aversion coefficient is not easy. For
} example, Kydland and Prescott [41] find that the relative risk aversion parameter is between 2 and 3 for most 
about the target returns, the dynamic mean variance policies in the existing literature either may be time inconsistent or contradict conventional investment wisdom, such that (i) rich people should invest more absolute amount of money in risky assets; (ii) the longer the investment horizon, the more proportional amount of money may be invested in risky assets; and (iii) for long-term investment, people should not short sell major stock indices whose returns are higher than the risk-free rate $2^{2}$

In this paper, we propose a dynamic portfolio choice model with mean-variance criterion for portfolio log-returns (hereafter log-MV criterion, for short), instead of with the standard mean-variance criteria for terminal wealth in the existing literature $3^{3}$ By combining certain advantages of both Markowitz's and Merton's models, our contribution is fourfold:

(1) In a complete market with constant market parameters, the model leads to an explicit formula for the optimal time-consistent mean-variance policy. There is a one-to-one mapping between the policy and the well-known Merton's myopic policy for constant relative risk aversion (CRRA) utilities in the complete market. Furthermore, similar to the singleperiod Markowitz model, we can elicit the mean-variance preference parameter by asking an investor to input the target annual return in the complete market; see Section 3 .

(2) The model is analytically tractable even under some incomplete market settings, and the resulting trading policies consists of familiar myopic and hedging components. In cases where analytical solutions are unavailable, we propose a link to some backward stochastic differential equations (BSDEs), which can be solved numerically to find the optimal time-consistent policies; see Section 4 .

(3) The model is capable of handling portfolio constraints such as the no-borrowing and no short-sale constraints; see Section 5 .

(4) Using the analytical solutions for the dynamic log-MV model in two special cases, a general Heston's stochastic volatility setting and a Gaussian mean return setting, we find that the optimal trading policies in both settings conform with the three criteria of the

investors; however, to explain the equity premium puzzle, the relative risk aversion parameter should be at least larger than 11 (e.g., Mehra and Prescott [48] and Mankiw and Zeldes [46]).

${ }^{2}$ Here we list the three investment criteria because they are widely accepted by the general public, and they are useful to serve as a basis to make a comparison with other dynamic mean-variance models in the literature.

${ }^{3}$ See, e.g., Bajeux-Besnainou and Portait [2], Basak and Chabakauri [4, Basak and Chabakauri [5], Björk, Murgoci, and Zhou [11, Cochrane [22], Li and $\mathrm{Ng}$ [42], and Zhou and $\mathrm{Li}$ [57]. 
conventional investment wisdom mentioned above; see, e.g., Propositions 1 and 2. We also find that our mean-variance portfolios mimic the corresponding CRRA portfolios under the above market settings; see Figures 5 and 6 , and compare the similarity between (23) and (25), and the similarity between (29) and (31).

\subsection{Literature Review: General Literature}

We study a dynamic portfolio choice problem with the log-MV criterion under general incomplete markets and obtain analytical solutions for two special settings, the general Heston's stochastic volatility and the time-varying Gaussian mean return. These two special settings have been widely studied with different objectives. For example, the expected utility maximization problems with the CRRA utility under the market settings are solved by Liu [43, 44] and Kim and Omberg [40], respectively. Basak and Chabakauri [4] consider a dynamic mean-variance criterion for terminal wealth under both of the market settings. Campbell and Viceira [17] study portfolio optimization with the Epstein-Zin recursive utility under the Gaussian mean return setting in discrete time. Some extensions are also studied by others, e.g. Chacko and Viceira [19] for the consumption and investment problem with a special stochastic volatility setting and recursive utility, and Wachter [55] for the consumption and investment problem with a special Gaussian mean return setting.

In this paper we choose to conduct mean-variance analysis, rather than the expected utility maximization, mainly because it is difficult or less straightforward to give an accurate estimate of the risk aversion parameter implied by utility functions. In fact, estimating the risk aversion parameter involved in the expected utility maximization theory is non-trivial even for professional people.

Many techniques have been suggested to mitigate the sensitivity of the mean-variance analysis to the parameter estimation. For example, shrinkage estimators, such as the JamesStein estimator, may be used; see, e.g, Jorion [39]. Alternatively, Jagannathan and Ma [38] show that imposing a short sale constraint is equivalent to shrinking the elements of the covariance matrix. One can use advanced big data techniques to estimate the covariance matrices and to impose short sale constraints; see, e.g., Fan, Zhang, and Yu [33], Fan, Liao, and Mincheva [32], Fan, Fan, and Lv [30], Fan, Liao, and Liu [31]. We do not study statistical estimation in this paper, but our model can incorporate portfolio constraints. 


\subsection{Literature Review: Dynamic Mean Variance Analysis}

The single-period mean-variance portfolio choice has been widely used in both academia and financial industry. Nevertheless, generalizing the mean-variance analysis to a dynamic setting (multi-period or continuous time) is usually challenging due to the inherent time inconsistency, namely that a mean-variance policy that is optimal today might not be optimal tomorrow. A simple way of handling this time inconsistency is to optimise the myopic mean-variance objective in each period, and roll up until maturity (e.g., Ait-Sahalia and Brandt [1]; Campbell and Viceira [18]). However, there is growing evidence that such a myopic policy is suboptimal in the presence of stochastic volatility or stochastic returns (e.g., Brandt [13]; Campbell and Viceira [17]). An alternative way is to seek a mean-variance strategy that is optimal at the initial time, and stick to the strategy until maturity 4 This strategy, known as the pre-committed strategy, disregards the sub-optimality in the future and leads to a time-inconsistent decision.

A breakthrough is made by Basak and Chabakauri [4 who obtain an explicit timeconsistent optimal policy for the mean-variance criterion for terminal wealth. In their model, the optimal dollar amount invested in stocks is, however, independent of investors' total wealth, which implies that both the rich and the poor should allocate the same dollar amount to stocks, similar to the Merton's strategy for constant absolute risk aversion (CARA) investors. Björk, Murgoci, and Zhou [11] introduce wealth-dependent preference into the dynamic mean-variance criterion for terminal wealth, and the resulting stock investment is proportional to investors' total wealth, which caters to the taste of CRRA investors. However, their policy suggests that, even with a positive stock risk premium, the dollar amount of the stock investment may be negative when the investment horizon is sufficiently large, which violates the investment rule that short sale of a "good" index is not recommended in the long run. In addition, their model needs to numerically solve an integral equation (thus lacking analytical tractability) even in a complete market with constant market parameters, which makes it difficult to handle a more general market setting.

Comparing to Björk, Murgoci, and Zhou [11, our model leads to a wealth dependent policy that conforms with the investment wisdom that short-sale is never optimal for a

${ }^{4}$ See, e.g., Li and Ng [42], Zhou and Li [57], Bielecki et al. [7], Cvitanic, Lazrak, and Wang [24], Dai, Xu, and Zhou [26] and references therein. 
"good" index. Moreover, our model is more tractable and is able to deal with various incomplete markets (e.g. the general Heston's stochastic volatility and the time-varying Gaussian mean return). As in the CRRA utility maximization case, our explicit optimal portfolio policy consists of familiar myopic and intertemporal terms, where the intertemporal term reflects the intertemporal hedging demand of an investor who faces stochastic volatility and stochastic returns. In particular, with reasonable parameter values, the stock investment is increasing with the investment horizon, which is consistent with the conventional financial wisdom that people with longer investment horizon should invest more in stock.

There are several reasons that we adopt the log-MV criterion in this paper. First, it inherits the spirit of Markowitz's one period mean-variance analysis which is essentially on portfolio returns. In a continuous time setting, it is natural to consider log-returns of portfolio, as returns are continuously compounded. In addition, Luenberger [45] provides an axiom justification of the one-period log-MV criterion. Second, in practice, fund managers keep track of their fund return because money tends to flow into (out of) the fund whose return is higher (lower) than the return of a benchmark index.5 Therefore, fund managers pursue a trade-off between high portfolio return and stability, which is perfectly captured by our criterion. Third, such a criterion naturally leads to a wealth-dependent strategy. In fact, our optimal trading strategy turns out to tally with the criteria of the conventional financial wisdom aforementioned, some of which are violated in previous mean-variance models. Table 1 summarizes a comparison of this paper versus some key papers.

Our model can handle portfolio constraints such as the no-borrowing and no short-sale constraints. In a complete market with portfolio constraints, our mean-variance policy is analytically available and is the same as that under the CRRA utility maximization (see, e.g., Cvitanic and Karazatzs [23] and Dai, Jin, and Liu [25]); the policy is myopic with respect to portfolio constraints in the sense that no action is taken before the constraints are binding. However, in incomplete markets, our mean-variance optimization is no longer myopic with respect to portfolio constraints.

It is also straightforward to extend our model to a multiple stocks setting. Moreover, in the absence of analytical solutions, we can employ either the finite difference method for ${ }^{5}$ See, e.g., Gruber [35], Brown, Harlow, and Starks [16], Chevalier and Ellison [21], Sirri and Tufano [53], and Basak and Makarov [6]. 
Table 1 A comparison of key literature

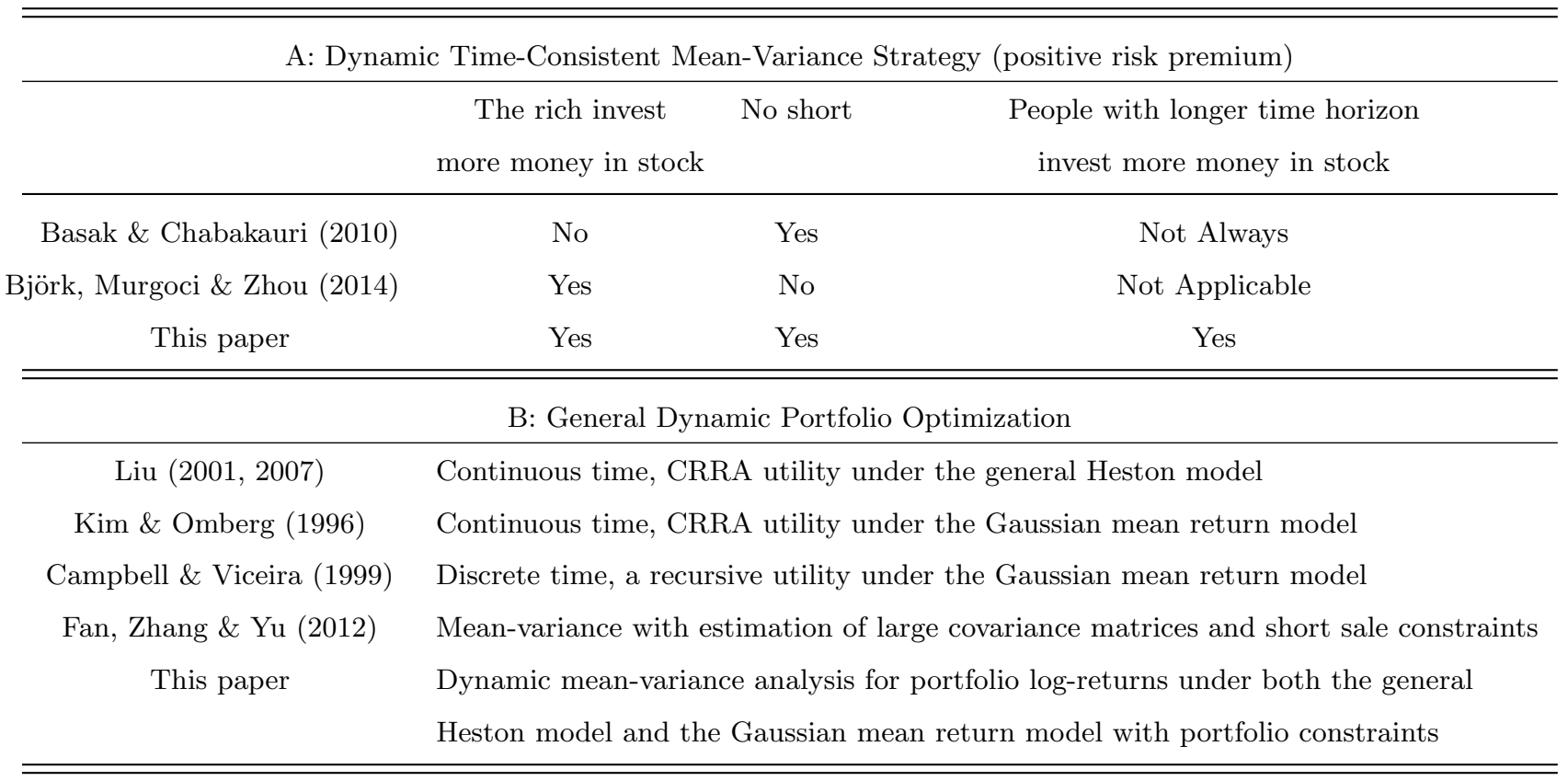

partial differential equations (PDEs) or Monte Carlo simulation for BSDEs to numerically find optimal policy; see Online Supplement A.

Technically our paper is connected to equilibrium solutions for dynamic decision making with time-inconsistency, dating back to 1950 's 6 - In comparison with this line of literature, our paper is the first one to consider the time consistency solution for the dynamic log-MV portfolio choice. Moreover, in terms of methodologies, we use the BSDE technique to prove the existence of equilibrium solutions and to find optimal policies; this appears to be new for dynamic mean variance.

The rest of the paper is organized as follows. In the next section, we present two dynamic $\log$-MV formulations and show their connection. In Section 3, we study the dynamic logMV portfolio choice in a complete market with constant market parameters and provide an intuitive way to identify the mean-variance preference parameter used in our model. Section 4 is devoted to the portfolio choice under general incomplete market settings. An extension of our model to incorporate portfolio constraints is given in Section 5. Section 6 concludes. An extension to the multiple risky assets case, a Monte-Carlo simulation approach for implementing our model, and all technical proofs are relegated to Online Supplement.

\footnotetext{
${ }^{6}$ See, e.g., Strotz [54], Peleg and Yaari [51], Ekeland and Lazrak [28], Ekeland and Pirvu [29], Björk, and Murgoci [9], and Björk, Khapko, and Murgoci [10]
} 


\section{Log Mean-Variance Criterion}

In this section, we present two formulations for dynamic log-MV criterion, and show the connection between the two formulations.

\subsection{Problem Setting}

We start with a market in which there are two assets available for investment: a riskless asset (bond) with interest rate $r_{t}$, and a risky asset (stock). The stock price evolves according to

$$
d S_{t}=\mu_{t} S_{t} d t+\sigma_{t} S_{t} d B_{t},
$$

where the drift rate $\mu_{t}$ and volatility $\sigma_{t}>0$, together with the interest rate $r_{t}$, are all adapted processes, and $B_{t}$ is a standard Brownian motion. A self-financing wealth process $W_{t}$ can be described by

$$
d W_{t}=\left[r_{t} W_{t}+\left(\mu_{t}-r_{t}\right) u_{t}\right] d t+\sigma_{t} u_{t} d B_{t}
$$

where $u_{t}$ is an adapted process representing the dollar amount invested in the stock at time $t$. In this paper, we do not allow investors to go bankrupt, i.e., $W_{t}>0$ almost surely. As a result, we can rewrite the wealth process as follows:

$$
\frac{d W_{t}}{W_{t}}=\left[r_{t}+\left(\mu_{t}-r_{t}\right) \pi_{t}\right] d t+\sigma_{t} \pi_{t} d B_{t},
$$

where $\pi_{t}:=u_{t} / W_{t}$, the fraction of the total wealth in the stock, stands for a trading strategy which is admissible if $\pi_{t}$ is adapted and $\mathbb{E}\left[\int_{0}^{T}\left|\sigma_{t} \pi_{t}\right|^{2} d t\right]<+\infty$.

Let $T$ be the investment horizon. Almost all of the existing literature on dynamic meanvariance portfolio choice focuses on the mean-variance criteria for terminal wealth $W_{T}$. In contrast, we propose a dynamic mean-variance criterion for log-return of the portfolio, that is, at any time $t<T$, we aim to maximize the objective

$$
\mathbb{E}_{t}\left[\ln \frac{W_{T}}{W_{t}}\right]-\frac{\gamma_{t}}{2} \operatorname{Var}_{t}\left[\ln \frac{W_{T}}{W_{t}}\right]
$$

by choosing an admissible strategy $\pi_{t}$, subject to (3), where $\mathbb{E}_{t}$ and $\operatorname{Var}_{t}$ represent the conditional expectation and the conditional variance at time $t$, respectively, and $\gamma_{t}>0$ can be regarded as the mean-variance preference parameter measuring the tradeoff between risk and return at time $t$. 
It should be emphasized that dynamic portfolio choice (4) is time-inconsistent, and we are concerned with time-consistent strategies under a certain sense of optimality 7 We will follow Björk, Khapko, and Murgoci [10] to introduce the concept of an equilibrium strategy to our dynamic mean-variance problem. In what follows, we assume an incomplete market setting with stochastic market parameters in which the riskfree rate, the stock return rate, and the stock volatility are all deterministic functions of time $t$ and a stochastic state variable $X_{t}$, namely, $r_{t}=r\left(t, X_{t}\right), \mu_{t}=\mu\left(t, X_{t}\right), \sigma_{t}=\sigma\left(t, X_{t}\right)$, and $X_{t}$ follows the dynamics:

$$
d X_{t}=m\left(t, X_{t}\right) d t+\nu\left(t, X_{t}\right) d B_{t}^{X},
$$

where $B_{t}^{X}$ is another standard Brownian motion correlated with $B_{t}$ by $\mathbb{E}\left[d B_{t}^{X} d B_{t}\right]=\rho d t$ with constant $\rho \in[-1,1]$, and $m(\cdot, \cdot)$ and $v(\cdot, \cdot)$ are two deterministic functions. All of these deterministic functions are assumed to be right continuous in $t$. Without extra effort, we can extend it to a more general case such as these deterministic functions depending on $S_{t}$ as well.

To simplify notation, we denote $R_{t}=\ln W_{t}$ which satisfies

$$
d R_{t}=\left[r_{t}+\left(\mu_{t}-r_{t}\right) \pi_{t}-\frac{1}{2} \sigma_{t}^{2} \pi_{t}^{2}\right] d t+\sigma_{t} \pi_{t} d B_{t} .
$$

Due to the well-known Markovian property implied by (6) and (5), we restrict attention to feedback strategy $\pi_{t}=\pi\left(t, R_{t}, X_{t}\right)$. The maximization problem with the objective function (4) can be rewritten as

$$
\max _{\pi \in \mathcal{A}_{t}} \mathbb{E}_{t}\left[R_{T}\right]-\frac{\gamma_{t}}{2} \operatorname{Var}_{t}\left[R_{T}\right] \quad t \in[0, T),
$$

where the set of admissible strategies $\mathcal{A}_{t}$ is defined as

$$
\mathcal{A}_{t}=\left\{\pi_{s}=\pi\left(s, R_{s}, X_{s}\right): \mathbb{E}_{t}\left[\int_{t}^{T}\left|\sigma_{s} \pi\left(s, R_{s}^{\pi}, X_{s}\right)\right|^{2} d s\right]<+\infty\right\} .
$$

To emphasize the dependence of the log-return process on $\pi$, we denote by $R_{t}^{\pi}$ the log-return process associated with $\pi$. The reward function related to $\pi$ is denoted by

$$
J\left(t, R_{t}, X_{t} ; \pi\right):=\mathbb{E}_{t}\left[R_{T}^{\pi}\right]-\frac{\gamma_{t}}{2} \operatorname{Var}_{t}\left[R_{T}^{\pi}\right] .
$$

\footnotetext{
${ }^{7}$ We point out that the dynamic mean-variance criterion for log-return does not yield an analytical solution for the optimal pre-committed policy even under the geometric Brownian motion model. In contrast, Zhou and Li [57] present the explicit optimal pre-committed policy for the dynamic mean-variance criterion for terminal wealth, where the optimal dollar amount in stock is an affine function of the current wealth level.
} 
Following Björk, Khapko, and Murgoci [10], we now define an equilibrium solution to problem (7), which leads to an equilibrium strategy that is optimal locally at any time given that the strategy will be followed in the future.

Definition 1. An admissible trading strategy $\hat{\pi}(\cdot, \cdot, \cdot)$ is called an (optimal) equilibrium strategy for problem (7) if, at any time $t$, for any admissible perturbation strategy $\pi^{h, v} \in \mathcal{A}$ defined by

$$
\pi^{h, v}(\tau, y, x)= \begin{cases}v, & \text { for } t \leq \tau<t+h \\ \hat{\pi}(\tau, y, x), & \text { for } t+h \leq \tau \leq T\end{cases}
$$

with any $h \in \mathbb{R}^{+}$and $v \in \mathbb{R}$, the reward function $J(t, y, x ; \hat{\pi})$ is locally better off, namely,

$$
\liminf _{h \rightarrow 0^{+}} \frac{J(t, y, x ; \hat{\pi})-J\left(t, y, x ; \pi^{h, v}\right)}{h} \geq 0 .
$$

The equilibrium value function $V$ generated by the equilibrium strategy $\hat{\pi}$ is thus defined as $V(t, y, x)=J(t, y, x ; \hat{\pi})$.

This definition implicitly imposes time-consistency in the sense that, at any time $t<T$, given the trading strategy in the future the investor will not deviate from her current trading strategy. It should be pointed out in a discrete-time setting, this kind of timeconsistency can also be achieved by imposing backward induction and a new optimization problem at any time $t$ (see, e.g., [9] and [52]). However, in a continuous-time setting, because of the lack of "the previous time spot" and "the next time spot", the backward induction constraint cannot be easily adopted.

It is worthwhile pointing out that unlike in Björk, Murgoci, and Zhou [11], our formulation (7) does not lead to a dependence on the wealth value at time $t$. Therefore, we do not need the technique developed by Björk, Khapko, and Murgoci [10] for handling such dependence. Instead, we develop a BSDE approach to solve problem (7) in a general market setting, due to the fact that the log wealth process involves a quadratic control variable. 
In the formulation (7), we need to prescribe the mean-variance preference parameter $\gamma_{t}$ that is less intuitive. Let us consider an alternative dynamic mean variance formulation loyal to the original (one-period) Markowitz's model:

$$
\min _{\pi \in \mathcal{A}_{t}} \operatorname{Var}_{t}\left(R_{T}\right), \quad \text { subject to } \frac{1}{T-t} \mathbb{E}_{t}\left[R_{T}-R_{t}\right] \geq \hat{a}_{t}
$$

where $\hat{a}_{t}$ is a predetermined adaptive process, representing the investor's expected annual target return at time $t$. The formulation (8) indicates that the investor dynamically minimizes her risk subject to a predetermined target annual return, $\hat{a}_{t}$, which may depend on the investment horizon and the realized sample path.

We can similarly define equilibrium solution to problem (8). Indeed, we only need to replace $J$ and $\mathcal{A}_{t}$ in Definition 11 by $\bar{J}\left(t, R_{t}, X_{t} ; \pi\right):=-\operatorname{Var}_{t}\left[R_{T}^{\pi}\right]$ and $\overline{\mathcal{A}}_{t}=\left\{\pi \in \mathcal{A}_{t}\right.$ : $\left.\frac{1}{T-s} \mathbb{E}_{s}\left[R_{T}^{\pi}-R_{s}^{\pi}\right] \geq \hat{a}_{s}, s \in[t, T)\right\}$, respectively.

Recently He and Jiang [36] independently study the formulation (8), but focus on myopic strategies, by considering only deterministic drift and volatility. In contrast, we have a more general setting, resulting in an extra non-myopic term (known as intertemporal hedging demand) in markets with stochastic coefficients; in addition, similar to Basak and Chabakauri [4] and Björk, Khapko, and Murgoci [10], our focus is the formulation (7), and the formulation (8) will be employed to mainly identify the mean-variance preference parameter in a complete market.

\subsection{Connection between Two Formulations}

We have an interesting link from the formulation (7) to the formulation (8).

TheOREm 1. Let $\hat{\pi}$ be an equilibrium policy to (7) and $R_{t}^{\hat{\pi}}$ be the associated optimal return. Then $\hat{\pi}$ must be an equilibrium policy to (8) with

$$
\hat{a}_{t}=\frac{1}{T-t} \mathbb{E}_{t}\left[R_{T}^{\hat{\pi}}-R_{t}^{\hat{\pi}}\right]
$$

In general, there is no easy connection for the reverse direction, i.e. from an equilibrium policy for (8) to that for (7). Throughout the rest of this paper (unless otherwise stated), we always make the following assumption, and mainly focus on the formulation (7) in which the investor's mean-variance preference is characterized by a constant $\gamma$. 
Assumption A: The mean-variance preference parameter $\gamma$ for an individual investor is a positive constant and remains unchanged.

A natural and challenging question is how to estimate the mean-variance preference parameter $\gamma$ that measures the trade-off between mean return and variance. It is wellknown that in the single-period mean-variance model, there exists a one-one mapping between $\gamma$ and the expected target return. As a consequence, one may instead request the investor to input a target return, from which one can infer $\gamma$. The advantage of this idea is that the target return is very intuitive to investors who are given the market information (e.g. the expected return level and variance level of risky assets, and the risk-free rate

level in the market). Moreover, investors do not need to know the formula between $\gamma$ and the target return, or to understand any optimization behind the mean-variance portfolio selection. Interestingly, as will be shown in (12), under the complete market with constant investment opportunity, our dynamic model also reveals a one-one mapping between $\gamma$ and the annual target return. This allows us to borrow the idea used in the single-period model to estimate $\gamma$ via a fictitious complete market. Thanks to Assumption A, the parameter $\gamma$ will be used to solve our mean-variance problem in an incomplete market.

\section{Portfolio Choices for a Complete Market}

In this section, we study the dynamic log-MV portfolio choice under a complete market setting with constant market parameters $\mu_{t} \equiv \mu, r_{t} \equiv r$, and $\sigma_{t} \equiv \sigma$.

\subsection{An Equilibrium Solution}

The portfolio choice problem with reward function (7) yields a closed form equilibrium solution in the complete market as follows.

THEOREM 2. Consider the mean-variance criterion (7) subject to (6) under the complete market setting with constant market parameters.

(i) An equilibrium strategy is given by

$$
\hat{\pi} \equiv \frac{\mu-r}{(1+\gamma) \sigma^{2}}
$$


(ii) The target annual return associated with the equilibrium strategy is constant, namely,

$$
\frac{1}{T-t} \mathbb{E}_{t}\left[R_{T}^{\hat{\pi}}-R_{t}^{\hat{\pi}}\right] \equiv \hat{a}=: r+\left(\frac{1}{1+\gamma}-\frac{1}{2} \frac{1}{(1+\gamma)^{2}}\right) \theta^{2}
$$

where $\theta=(\mu-r) / \sigma$. Moreover, $\hat{a} \in\left(r, r+\frac{1}{2} \theta^{2}\right)$.

Basak and Chabakauri [4 link a certain dynamic mean variance asset allocation to CARA preferences, generalizing the well-known connection between mean variance and utility maximization in a one-period setting. In contrast, part (i) of the above theorem establishes an equivalence between dynamic mean-variance and CRRA preferences in the complete market. More precisely, the dynamic mean-variance equilibrium strategy as given by (9) is the same as the optimal solution for CRRA utility maximization (Merton [49])

$$
\max _{\pi} \mathbb{E}_{t}\left[\frac{W_{T}^{1-\tilde{\gamma}}-1}{1-\tilde{\gamma}}\right]
$$

subject to the self-finance process (3), where the relative risk aversion parameter of the CRRA optimizer $\tilde{\gamma}=1+\gamma$. Note that CRRA utility is effectively a moment generating function of the $\log$ return:

$$
\begin{aligned}
\mathbb{E}_{t}\left[\frac{W_{T}^{1-\tilde{\gamma}}-1}{1-\tilde{\gamma}}\right] & =\mathbb{E}_{t}\left[\frac{e^{(1-\tilde{\gamma}) \ln W_{T}}-1}{1-\tilde{\gamma}}\right]=\mathbb{E}_{t}\left[\frac{1-e^{-\gamma \ln W_{T}}}{\gamma}\right] \\
& =\mathbb{E}_{t}\left[\ln W_{T}-\frac{\gamma}{2}\left(\ln W_{T}\right)^{2}+\frac{\gamma^{2}}{3 !}\left(\ln W_{T}\right)^{3}-\frac{\gamma^{3}}{4 !}\left(\ln W_{T}\right)^{4}+\cdots\right]
\end{aligned}
$$

which indicates that CRRA preferences make use of all moments of log-return, whereas mean-variance uses only the first two 8 It is easy to see that when $\gamma \rightarrow 0(\tilde{\gamma} \rightarrow 1)$, the meanvariance optimization and the CRRA utility maximization are identical and thus yield the same optimal solution. For $\gamma>0(\tilde{\gamma}>1)$, the two optimization problems have different implications, and the CRRA utility maximization is naturally time consistent whereas the mean-variance optimization is not. However, they share the same optimal strategy, despite that the optimality is achieved in different senses. Notice that the equivalence holds only for $\gamma>0$ and the mean-variance optimization does not make sense for $\gamma<0$, which coincides with the fact that the estimated value of the CRRA parameter $\tilde{\gamma}$ is usually larger than 1 .

${ }^{8}$ Based on this observation, the risk sensitive asset management model, e.g. in Bielecki and Pliska [8] and Davis and Lleo [27, is also relevant to our dynamic mean-variance model. However, there is no literature on estimating the risk aversion parameter in the risk sensitive asset management model. 
Later we will see that in incomplete markets, our dynamic mean-variance optimization and the CRRA utility maximization are not equivalent in general; instead, they are linked via a measure transformation. See Online Supplement ??. Since (11) remains valid in any markets, it is not surprising that our mean-variance policy still mimics the Merton's policy with CRRA preferences in incomplete markets; see Figures 5 and 6 in Section 4.

Part (ii) of Theorem 2 reveals that under a complete market setting with constant market parameters, the expected annual return of the equilibrium strategy is constant. It is easy to see that the return is monotonically decreasing with $\gamma$, which coincides with the intuition that the higher the mean-variance preference parameter, the lower the expected target return. Part (ii) also suggests that the mean-variance maximizer cannot expect an

unreasonable target return: At time $t$, any target return that is higher than $r+\frac{1}{2} \theta^{2}$ is never attainable.

\subsection{Recovery of mean-variance preference}

Note that 10 can be rewritten as

$$
\gamma=\frac{1}{1-\sqrt{1-2(\hat{a}-r) / \theta^{2}}}-1,
$$

which indicates a one-one mapping between the mean-variance preference parameter $\gamma$ and the expected target annual return $\hat{a}$. By Theorem 2, we infer the following result.

Corollary 1. Assume a complete market setting with constant market parameters. Consider the mean-variance criterion (8) with constant target annual return $\hat{a}_{t} \equiv \hat{a} \in(r, r+$ $\left.\frac{1}{2} \theta^{2}\right]$. Then an equilibrium strategy $\hat{\pi}$ is given by (9) and $\gamma$ is as given by (12).

With constant target annual return, problem (8) is intuitively more appealing. The above corollary indicates that under a complete market setting with constant market parameters, problem (8) with a given constant target annual return $\hat{a}$ shares the same equilibrium policy as problem (7) with constant mean-variance preference parameter $\gamma$ computed by (12). This observation suggests a simple and intuitive way, which can be employed to identify the mean-variance preference parameter $\gamma$ used in our dynamic mean-variance criterion (7): Given a "fictitious" complete market with (exogenously given) constant parameters $\mu$, sigma and $r$, an individual investor is asked in a questionnaire to input his/her constant 
target annual return $\hat{a}$; then the investor's mean-variance preference parameter $\gamma$ used in (7) can be identified through 12 .

The one-to-one mapping in 12 between $\hat{a}$ and $\gamma$ is special for the complete market with constant market parameters $(\mu, r, \sigma)$. In an incomplete market or a complete market with time-varying market parameters, in which investment opportunity is varying, investors are unlikely to maintain a constant annual target return $\hat{a}$; see, e.g., (19). As a result it may be difficult to infer investors' mean-variance preference parameter via their target returns. That is why we propose a fictitious complete market in the questionnaire to recover the preference parameter; and then, thanks to Assumption A, we can use the parameter $\gamma$ to solve the dynamic mean-variance problem in incomplete markets.

It should be pointed out that as in the single-period Markowitz framework, the estimation suffers a drawback: it may not be robust in the sense that a change in the expected target return may lead to a different $\gamma$. To partially overcome this disadvantage, we may repeat the estimation with several sets of market parameters and provide an average estimate of $\lambda$.

More precisely, in practice we can show investors in the questionnaire several constant investment environments, e.g. $\left(\mu_{i}, r_{i}, \sigma_{i}\right), i=1,2,3,4$, and ask investors to input their target returns $\hat{a}_{i}$ within the range between $r_{i}$ and $r_{i}+\frac{1}{2} \theta_{i}^{2}, i=1,2,3,4$, respectively. Then we can get $\gamma_{i}, i=1,2,3,4$ using 12 and recover bounds on the mean-variance preference parameter. Note that estimating the mean-variance preference parameter is a special case of estimating the risk profile of an investor. In general, how to estimate the risk profile in a robust way is a difficult problem in decision science.

Figure 1 plots the magnitude of $\gamma$ against the target return level $\hat{a}$ for given parameter values $r=0.02, \mu=0.08$, and $\sigma=0.15$. This figure shows that the portfolio's annual target return input by an investor implies his risk aversion degree in the complete market. For example, given the market parameter values, an annual target return $\hat{a}=0.06$ corresponds to the risk aversion parameter $\gamma=2.5$ in our model. Observe that $\gamma$ is monotonically decreasing as $\hat{a}$ increases, which implies that the more risk averse people are, the lower the annual return level they target. In addition, Theorem 2 indicates that the target return does not exceed a certain range, which is also verified by Figure 1. 


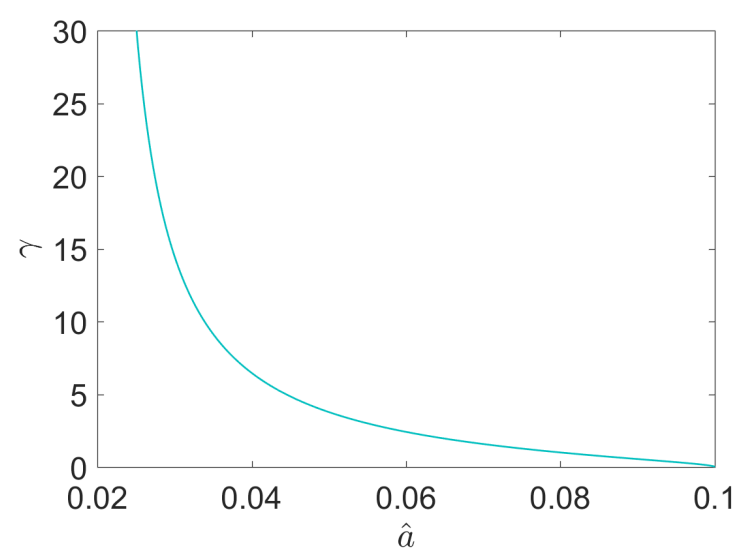

Figure 1 The change of the mean-variance preference parameter $\gamma$ against the target annual return $\hat{a}$ under the complete market setting with constant market parameters. Default parameter values: $r=0.02, \mu=0.08$, $\sigma=0.15$. This figure indicates that the portfolio's annual return that an investor targets implies his risk aversion degree. For example, given the market parameter values, an annual target return $\hat{a}=0.06$ corresponds to the risk aversion parameter $\gamma=2.5$ in our model.

\subsection{A Comparison of Different Strategies}

We shall compare our model with two existing dynamic mean-variance models that also seek equilibrium solutions. Basak and Chabakauri [4] study the following dynamic meanvariance criterion for terminal wealth, namely,

$$
\mathbb{E}_{t}\left[W_{T}\right]-\frac{\gamma}{2} \operatorname{Var}_{t}\left(W_{T}\right)
$$

subject to (2), where $\gamma$ is constant, and bankruptcy is permitted. In the complete market, they find that an equilibrium strategy is to invest the following dollar amount in the stock:

$$
\frac{\mu-r}{\gamma \sigma} e^{-r(T-t)}
$$

which indicates that as in the case of CARA utility maximization, the investment policy is independent of current wealth. The policy is economically less reasonable because the rich and the poor should not have the same investment strategy.

Björk, Murgoci, and Zhou [11] propose a dynamic mean-variance criterion with wealthdependent mean-variance preference described as follows:

$$
\mathbb{E}_{t}\left[W_{T}\right]-\frac{\gamma}{2 W_{t}} \operatorname{Var}_{t}\left(W_{T}\right)
$$


Assuming that $r_{t}, \mu_{t}$, and $\sigma_{t}$ are all constant, they find a wealth-dependent optimal strategy, where the dollar amount invested in the stock at time $t$ is $\pi_{*}(t) W_{t}$, with $\pi_{*}(t)$ being the unique solution to the integral equation

$$
\pi_{*}(t)=\frac{\mu-r}{\gamma \sigma^{2}}\left\{e^{-\int_{t}^{T}\left[r+(\mu-r) \pi_{*}(s)+\sigma^{2} \pi_{*}^{2}(s)\right] d s}+\gamma e^{-\int_{t}^{T} \sigma^{2} \pi_{*}^{2}(s) d s}-\gamma\right\}
$$

Since the integral equation does not permit analytical solutions, one has to rely on numerical solutions even in the complete market with constant market parameters, which makes it difficult to handle a more general market setting.

Numerical results show that with a shorter investment horizon, the integral equation indeed permits a unique positive solution for positive risk premium $\mu-r>0$. Unfortunately, it is easy to see

$$
\lim _{T-t \rightarrow+\infty} \pi_{*}(t)=-\frac{\mu-r}{\sigma^{2}}
$$

which suggests that in the long run, one needs to short sell a "good" stock (namely, a stock with positive risk premium). This result is somewhat counter-intuitive, since short sale of a major index is rare for a long-term investment. In contrast, our model not only generates a economically reasonable trading policy but also is analytically more tractable: it can be seen later that our model permits analytical solutions even in many incomplete markets.

Table 2 summarizes key differences between our model and the models of Basak and Chabakauri [4] and Björk, Murgoci, and Zhou [11]. It is easy to verify that our model possesses nice properties consistent with conventional investment rules. For example, 1) our model can deal with the no-bankruptcy constraint (see Section 5.1); 2) Given a positive stock risk premium, one should not short sell the stock with positive risk premium, i.e. a stock with $\mu>r$; and 3) the dollar amount invested in the stock is monotonically increasing in wealth and stock return, which implies that the rich invest more dollar amount in the stock than the poor do, and that the higher the stock risk premium, the more the investment in the stock. The optimal polices of Basak and Chabakauri [4] and Björk, Murgoci, and Zhou [11] may violate some of these desirable properties.

Figure 2 plots the dollar amounts invested in the stock against total wealth at time 0 for the three strategies with parameter values $\gamma=1, \sigma=0.17, \mu=0.08, r=0.03$, and $T=30$. It can be observed that given the parameter values, the policy of Björk, Murgoci, 
Table 2 A comparison of optimal strategies for dynamic mean-variance models $(\mu>r)$

\begin{tabular}{clccc}
\hline & $\begin{array}{l}\text { optimal dollar } \\
\text { amount in stock }\end{array}$ & bankruptcy & short sale & $\begin{array}{c}\text { monotonicity of stock } \\
\text { investment in } W_{t} \text { and } \mu\end{array}$ \\
\hline Basak-Chabakauri & $\frac{\mu-r}{\gamma \sigma^{2}} e^{-r(T-t)}$ & possible & never & only in $\mu$ \\
\hline Björk-Murgoci-Zhou & $\begin{array}{l}\pi_{*}(t) W_{t}, \text { with } \pi_{*}(t) \\
\text { given in } \sqrt{13}\end{array}$ & never & possible & $\begin{array}{c}\text { depending on } \\
\text { investment horizon }\end{array}$ \\
\hline This paper & $\frac{\mu-r}{(1+\gamma) \sigma^{2}} \cdot W_{t}$ & never & never & both \\
\hline
\end{tabular}

and Zhou [11] is to short-sell stock even with the positive risk premium $\mu-r=0.05$, the dollar amount in stock for Basak and Chabakauri [4] is independent of wealth, and our strategy is to keep a constant (positive) fraction of total wealth in stock.

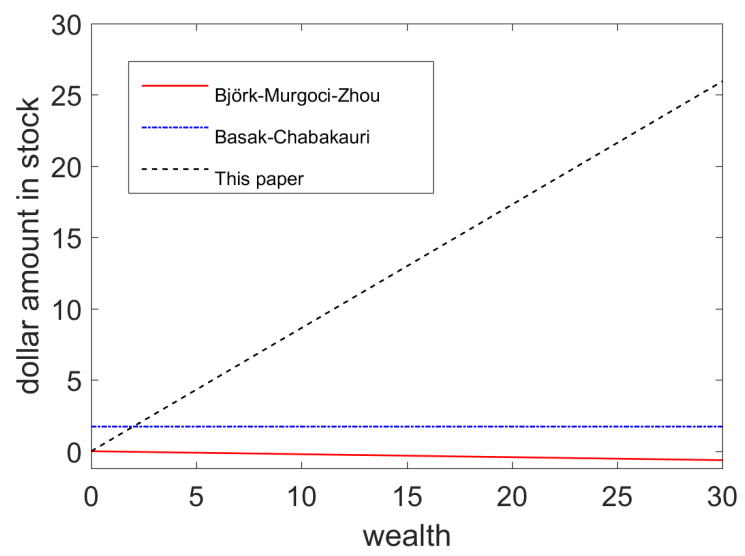

Figure 2 The dollar amounts invested in stock against the dollar amount of total wealth for our model and the models of Basak and Chabakauri [4] and Björk, Murgoci, and Zhou [11], respectively, under the complete market setting with constant market parameters. Parameter values: $\gamma=1, \sigma=0.17, \mu=0.08, r=0.03, T=30$. It can be seen that given the parameter values, the policy of Björk, Murgoci, and Zhou [11] is to short-sell stock even with the positive risk premium $\mu-r=0.05$, the dollar amount in stock for Basak and Chabakauri [4] is independent of wealth, while our policy is to hold $86.5 \%$ of total wealth in stock.

\section{Portfolio Choices for Incomplete Markets}

In this section, we consider the dynamic log-return mean-variance criterion under incomplete market settings. We will show that the model is still analytically tractable in some cases. Moreover, given reasonable market parameters, the resulting investment policies also comply with the popular investment advice mentioned earlier. 


\subsection{A General Theorem}

It turns out that under the incomplete market settings as described by (1) and (5), a semi-analytical equilibrium solution is still available, and the corresponding percentage allocation to the stock $\hat{\pi}_{t}=\hat{\pi}\left(t, X_{t}\right)$ is independent of $R_{t}$. The result is stated in the following.

THEOREM 3. Consider the mean-variance criterion (7) subject to (6) under the incomplete market setting (1) and (5). Assume

$$
\mathbb{E}\left[\int_{0}^{T} \theta_{s}^{2} d s\right]<+\infty, \quad \mathbb{E}\left[e^{-\frac{\gamma^{2} \rho^{2}}{(1+\gamma)^{2}}\left(\int_{0}^{T}\left(r_{t}+\theta_{t}^{2} / 2\right) d t+\int_{0}^{T} \theta_{t} / \rho d B_{t}^{X}\right)}\right]<+\infty,
$$

where $\theta_{t}=\theta\left(t, X_{t}\right)=\frac{\mu\left(t, X_{t}\right)-r\left(t, X_{t}\right)}{\sigma\left(t, X_{t}\right)}$. Then we have the following results.

(i) An equilibrium policy is given by

$$
\hat{\pi}\left(t, X_{t}\right)=\frac{\mu_{t}-r_{t}}{(1+\gamma) \sigma_{t}^{2}}-\frac{\rho \gamma Z_{t}}{(1+\gamma) \sigma_{t}}
$$

where $Z_{t}$ is uniquely determined by the BSDE

$$
d Y_{t}=-g\left(t, Z_{t}\right) d t+Z_{t} d B_{t}^{X}, \quad Y_{T}=0
$$

with $g(t, z)=r_{t}+\frac{1}{2} \theta_{t}^{2}-\frac{\gamma^{2}\left(\theta_{t}+\rho z\right)^{2}}{2(1+\gamma)^{2}}$.

(ii) For the non-trivial case $\rho \neq 0$, there exists a deterministic function $f(\cdot, \cdot)$ such that the solution $(Y, Z)$ for the BSDE (15) satisfies

$$
\begin{aligned}
Y_{t} & =f\left(t, X_{t}\right)=:-\frac{(1+\gamma)^{2}}{\gamma^{2} \rho^{2}} \ln \mathbb{E}_{t}\left[e^{-\frac{\gamma^{2} \rho^{2}}{(1+\gamma)^{2}}\left(\int_{t}^{T}\left(r_{s}+\frac{\theta_{s}^{2}}{2}\right) d s+\int_{t}^{T} \theta_{s} / \rho d B_{s}^{X}\right)}\right], \\
Z_{t} & =\nu\left(t, X_{t}\right) \frac{\partial f}{\partial x}\left(t, X_{t}\right) .
\end{aligned}
$$

Moreover, if $f(\cdot, \cdot)$ is $C^{1,2}$, then $f(\cdot, \cdot)$ solves

$$
\left\{\begin{array}{l}
\frac{\partial f}{\partial t}+m(t, x) \frac{\partial f}{\partial x}+\frac{\nu^{2}(t, x)}{2} \frac{\partial^{2} f}{\partial x^{2}}+g\left(t, \nu(t, x) \frac{\partial f}{\partial x}\right)=0 \\
f(T, x)=0
\end{array}\right.
$$


Furthermore,

$$
\frac{1}{T-t} \mathbb{E}_{t}\left[R_{T}^{\hat{\pi}}-R_{t}^{\hat{\pi}}\right]=\frac{1}{T-t} f\left(t, X_{t}\right), t \in[0, T)
$$

Part (i) of the above theorem shows that the equilibrium policy consists of two terms. The first term, $\frac{\mu_{t}-r_{t}}{(1+\gamma) \sigma_{t}^{2}}$, corresponds to the myopic demand fully determined by the current values of market parameters, which is the same as the equilibrium policy obtained in a complete market with constant market parameters. The second term, $-\frac{\rho \gamma Z_{t}}{(1+\gamma) \sigma_{t}}=-\frac{\rho \gamma \nu t}{(1+\gamma) \sigma_{t}} \frac{\partial f}{\partial x}$, is often referred to as the intertemporal hedging demand (Merton [49], Basak and Chabakauri [4]), which is to hedge against the fluctuations in exogenous random investment opportunities. Note that the hedging demand vanishes when the correlation $\rho=0$, as there is no feasible way of hedging.

Part (ii) of the above theorem presents a semi-explicit equilibrium policy in a general incomplete market, which provides two numerical ways to find the optimal policies, either via the Monte Carlo simulation for the expectation expression (16) or via the finite difference method for PDE (18). Note that the expectation expression (16) can be extended to the multi-asset case provided the stochastic state variable $X_{t}$ is one-dimensional; when $X_{t}$ is multi-dimensional, such an expectation expression is no longer available, but we are still able to use Monte-Carlo simulations for BSDEs to numerically find equilibrium policy. This is because our problem can be formulated as BSDEs and there exist efficient MonteCarlo simulations for BSDEs in literature (e.g., Chassagneux and Richou [20]). In Online Supplement ??, we show how to employ the Monte-Carlo simulation approach developed by Chassagneux and Richou [20] to compute $f(t, x)$ and its derivatives.

In contrast to the invariant annual target return in a complete market with constant market parameters, 19 indicates that in an incomplete market, the target annual return associated with the equilibrium solution depends on the stochastic variable $X_{t}$ as well as the time to maturity, which is consistent with the intuition that the investor's expected return may change subject to market performance and investment horizon. Unlike a complete market with constant parameters in which investment opportunity is invariant, an incomplete market leads to time-varying investment opportunity. Consequently, specifying an invariant (i.e. independent of time or state variables) annual target return may make no sense. Therefore, it is impossible to elicit investors' mean-variance preference parameter $\gamma$ in an incomplete market by only use of an invariant annual target return. That is 
why we resort to a fictitious complete market with constant investment opportunity in the questionnaire to recover investors' risk preferences.

When directly considering the mean-variance problem (8) in incomplete markets, we face a difficulty of specifying reasonable dynamic annual target return $\hat{a}_{t}$. To mitigate the difficulty, noting that Theorem 1 also applies to incomplete markets, we conclude from Theorem 1 and Theorem 3(ii) that the equilibrium solution as given in (14) for the meanvariance problem (7) with constant mean-variance preference parameter also solves the mean-variance problem (8) with a time-varying and state-dependent annual target return $\hat{a}_{t}=\frac{1}{T-t} f\left(t, X_{t}\right)$, where $f(\cdot, \cdot)$ is as given by $(16)$. Due to the difficulty in specifying $\hat{a}_{t}$ in (8) for incomplete markets, this paper only focuses on the mean-variance problem (7) for incomplete markets.

Although in incomplete markets the mean-variance criterion for wealth is different from the CARA-type utility maximization, Basak and Chabakauri [4] show that their optimal equilibrium strategy is identical to the policy obtained by a CARA-type utility maximization through a change of probability measure. We can derive a similar result that links our mean-variance criterion for log returns to CRRA preferences. Indeed, let $\hat{\pi}$ be the equilibrium strategy as given in (14). Define the random variable $\varepsilon$ by $\varepsilon=e^{-\frac{\gamma^{2}}{2} \int_{0}^{T} \operatorname{Var}_{t}\left(d \mathbb{E}_{t}\left[R_{T}^{\hat{x}}\right]\right)}$, where $\operatorname{Var}_{t}\left(d E_{t}\left[R_{T}^{\hat{\pi}}\right]\right)$ is the conditional variance of the local increment of $\left.\left.E_{t}\left[R_{T}^{\hat{\pi}}\right]\right]\right]^{9}$ Since $\varepsilon>0$, we can construct a new probability measure $\mathbb{Q}$ by the density $\frac{\varepsilon}{\mathbb{E}[\varepsilon]}$. Then the strategy (14) is the same as the optimal policy for the CRRA utility maximization $\max _{\pi} \mathbb{E}_{\mathbb{Q}}\left[\frac{W_{T}^{1-\tilde{\gamma}}}{1-\tilde{\gamma}}\right]$ with $\tilde{\gamma}=1+\gamma$. A detailed proof is in Online Supplement ??.

\subsection{Analytical Solutions for Two Cases}

The PDE problem (18) usually does not admit analytical solutions for a general process of $X_{t}$. However, some special structures in $X_{t}$ may lead to explicit equilibrium solutions and allow us to explore the implications of dynamic mean-variance investment policies for markets with stochastic coefficients.

4.2.1. Stochastic Volatility Consider a stochastic volatility model in which the stock price $S_{t}$ and a state variable $X_{t}$ follow

$$
\frac{d S_{t}}{S_{t}}=\left(r+\delta X_{t}^{\frac{1+\alpha}{2 \alpha}}\right) d t+X_{t}^{\frac{1}{2 \alpha}} d B_{t}
$$

${ }^{9}$ The notation $\int_{0}^{T} \operatorname{Var}_{t}\left(d \mathbb{E}_{t}\left[R_{T}^{\hat{\pi}}\right]\right)$ is not mathematically rigorous. A rigorous notation can be used, if we denote $Z_{t}=\mathbb{E}_{t}\left[R_{T}^{\hat{\pi}}\right]$, then $\int_{0}^{T} \operatorname{Var}_{t}\left(d \mathbb{E}_{t}\left[R_{T}^{\hat{\pi}}\right]\right)=\langle Z\rangle_{T}$ is the quadratic variation of the process $Z$ over the time interval $[0, T]$. 


$$
d X_{t}=\lambda\left(\bar{X}-X_{t}\right) d t+\bar{\nu} \sqrt{X_{t}} d B_{t}^{X},
$$

where $\alpha \neq 0$ is the constant elasticity of the market price of risk $\delta X_{t}^{\frac{1}{2}}, \delta \in \mathbb{R}, \lambda>0, \bar{\nu}>0$, and $\bar{X} \in \mathbb{R}$ are all constants. Dynamic portfolio choice under this market setting has been widely studied. See, e.g., Liu [43] for CRRA preferences for the terminal wealth, Chacko and Viceira [19] for recursive preferences of intermediate consumption (the case $\alpha=-1$ ), and Basak and Chabakauri [4] for the mean-variance preferences for terminal wealth.

Proposition 1. An equilibrium investment policy for the stochastic volatility market (20) - 21) is given by

$$
\hat{\pi}\left(t, X_{t}\right)=\frac{\delta}{1+\gamma} X_{t}^{\frac{\alpha-1}{2 \alpha}}+\hat{H}\left(t, X_{t}\right)
$$

where

$$
\begin{aligned}
& \hat{H}\left(t, X_{t}\right)=-\frac{\iota \gamma \rho \bar{\nu} \delta^{2}}{(1+\gamma)^{2}} X_{t}^{\frac{\alpha-1}{2 \alpha}} \frac{e^{\bar{q}(T-t)}-1}{(\bar{b}+\bar{q})\left(e^{\bar{q}(T-t)}-1\right)+2 \bar{q}}, \\
& \iota=\frac{1+2 \gamma}{1+\gamma}, \quad \bar{q}=\frac{1}{1+\gamma}\left[\gamma^{2}(\lambda+\rho \bar{\nu} \delta)^{2}+\lambda^{2}(2 \gamma+1)\right]^{\frac{1}{2}}, \quad \bar{b}=\lambda+\frac{\gamma^{2} \rho \delta \bar{\nu}}{(1+\gamma)^{2}}
\end{aligned}
$$

Consequently,

(i) the optimal percentage allocation to the stock $\hat{\pi}\left(t, X_{t}\right)$ is positive (negative) for $\delta>0$ $(\delta<0)$

(ii) $\hat{\pi}\left(t, X_{t}\right)$ is increasing (decreasing) in the market price of risk $\delta X_{t}^{\frac{1}{2}}$ when $\alpha<0$ or $\alpha>1(\alpha \in(0,1)), 10$

(iii) The hedging demand $\hat{H}\left(t, X_{t}\right)$ is positive (negative) when $\rho<0(\rho>0)$;

(iv) $\hat{\pi}\left(t, X_{t}\right)$ is increasing (decreasing) in the investment horizon $T-t$ when $\rho<0(\rho>0)$.

\footnotetext{
${ }^{10}$ It can be seen immediately that $\hat{\pi}$ is independent of $X_{t}$ when $\alpha=1$, i.e. in the case of Heston's stochastic volatility model.
} 
Proposition 1 presents a percentage allocation rule. As is common, the optimal percentage allocation to the stock comprises familiar myopic and intertemporal hedging terms. Part (i) of Proposition 1 shows that for a "good" stock with positive risk premium (i.e. $\delta>0$ ), the optimal policy is to keep a certain positive fraction of total wealth to the stock, which generalizes the similar result under a complete market setting with constant market parameters. The result $\hat{\pi}>0$ for $\delta>0$ has two implications consistent with financial advice. First, the mean-variance optimizer should not short sell a "good" stock. Second, the rich should invest more dollar amount in stock than the poor, because the optimal percentage allocation $\hat{\pi}$ does not depend on wealth.

Part (ii) shows that the stock investment policy is increasing in the market price of risk when the elasticity of market price of risk with respect to stock volatility is either negative $(\alpha<0)$ or relatively high $(\alpha>1)$. Part (iii) indicates that the sign of the hedging demand depends on the sign of the correlation between the stock and the state variable. A negative correlation induces a positive hedging demand to offset the fluctuation risk due to stochastic investment opportunities. Similar properties and implications are presented by Basak and Chabakauri [4], though their investment policy is independent of current wealth.

Part (iv) of Proposition 1 characterizes the monotonicity of the the stock investment with respect to the investment horizon.

The case of $\alpha=1$ corresponds to the well-known Heston's stochastic volatility model for which empirical studies support a negative correlation between stochastic volatility and stock return (see [37]). We then deduce that in a market driven by the Heston model, people having longer investment horizon should invest more in stock, which is consistent with conventional financial wisdom. It should be pointed out that the optimal policy of Basak and Chabakauri [4] may not be monotone in the investment horizon (see Part (iv) in Corollary 2 in their paper).

Our model also suggests an asymptotic long-term investment policy, as $T-t \rightarrow \infty$ in (22), $\hat{\pi}_{\infty}\left(t, X_{t}\right)$, where

$$
\hat{\pi}_{\infty}\left(t, X_{t}\right)=\frac{\delta}{1+\gamma} X_{t}^{\frac{\alpha-1}{2 \alpha}}-\frac{\iota \gamma \rho \bar{\nu} \delta^{2}}{(1+\gamma)^{2}} X_{t}^{\frac{\alpha-1}{2 \alpha}} \frac{1}{\bar{b}+\bar{q}}
$$

with $\iota, \bar{b}$, and $\bar{q}$ defined by (23). Note that $\hat{\pi}_{\infty}\left(t, X_{t}\right)=0$ in Basak and Chabakauri [4]. 
As mentioned earlier, our mean-variance optimization problem takes the first two moments of the log return, while the CRRA utility maximization uses all moments. In a complete market, the two problems yield the same optimal investment policy. However, in an incomplete market, their policies differ due to different hedging demands. Interestingly, their hedging demands possess the same structure for this stochastic volatility market setting. Indeed, assuming CRRA risk aversion $\tilde{\gamma}=\gamma+1$, the optimal CRRA investment policy takes the same form as in (22) except that (23) is replaced by (see Liu [43])

$$
\iota=1, \quad \bar{q}=\left[\lambda^{2}+\frac{\gamma}{1+\gamma}\left(2 \lambda \rho \bar{\nu} \delta+\delta^{2} \bar{\nu}^{2}\right)\right]^{\frac{1}{2}}, \quad \bar{b}=\lambda+\frac{\gamma \rho \delta \bar{\nu}}{1+\gamma} .
$$

4.2.2. Time-Varying Gaussian Mean Return Consider the time-varying Gaussian mean return model in which the stock price $S_{t}$ and the market price of risk $X_{t}$ are governed by

$$
\begin{aligned}
\frac{d S_{t}}{S_{t}} & =\left(r+\sigma X_{t}\right) d t+\sigma d B_{t}, \\
d X_{t} & =\lambda\left(\bar{X}-X_{t}\right) d t+\nu d B_{t}^{X},
\end{aligned}
$$

where $r, \sigma, \lambda, \nu$, and $\bar{X}$ are all positive constants ${ }^{11}$ Dynamic portfolio choice under this market setting or its special case has also been widely studied by Merton [49], Kim and Omberg [40], Campbell Viceira [17], Wachter [55], and Basak and Chabakauri [4]. Note that all these literature, except Basak and Chabakauri [4], consider utility maximization. Here, as in Basak and Chabakauri [4], we consider the mean-variance type problem.

Proposition 2. An equilibrium policy for the time-varying Gaussian mean return market $26-(27)$ is given by

$$
\hat{\pi}\left(t, X_{t}\right)=\frac{X_{t}}{\sigma(1+\gamma)}+\hat{H}\left(t, X_{t}\right)
$$

where

$$
\begin{aligned}
\hat{H}\left(t, X_{t}\right) & =-\frac{\iota \gamma \rho \nu}{(\gamma+1)^{2} \sigma} \frac{q\left(e^{2 q(T-t)}-1\right) X_{t}+\lambda\left(e^{q(T-t)}-1\right)^{2} \bar{X}}{q\left[(b+q)\left(e^{2 q(T-t)}-1\right)+2 q\right]}, \\
\iota & =\frac{1+2 \gamma}{1+\gamma}, \quad q=\frac{1}{\gamma+1}\left[\gamma^{2}(\lambda+\rho \nu)^{2}+\lambda^{2}(2 \gamma+1)\right]^{\frac{1}{2}}, \quad b=\lambda+\frac{\gamma^{2} \rho \nu}{(1+\gamma)^{2}} .
\end{aligned}
$$

\footnotetext{
${ }^{11}$ For late analysis, we always assume $\mathbb{E}\left[X_{t}\right]=\bar{X}$ for all $t$. That is, $\bar{X}$ is the statistic mean value of $X_{t}$.
} 


\section{Consequently,}

(i) the optimal percentage allocation to the stock $\hat{\pi}\left(t, X_{t}\right)$ is increasing in the market price of risk $X_{t}$;

(ii) The optimal mean percentage allocation to the stock $\mathbb{E}\left[\hat{\pi}\left(t, X_{t}\right)\right]$ is positive (negative) when average market price of risk $\bar{X}$ is positive (negative);

(iii) The mean hedging demand $\mathbb{E}\left[\hat{H}\left(t, X_{t}\right)\right]$ is positive (negative) for $\rho<0(\rho>0)$ when $\bar{X}>0$, and the converse is true for $\bar{X}<0$;

(iv) The optimal mean percentage allocation to the stock $\mathbb{E}\left[\hat{\pi}\left(t, X_{t}\right)\right]$ is increasing (decreasing) in the investment horizon for $\rho<0(\rho>0)$ when $\bar{X}>0$, and the converse is true for $\bar{X}<0$.

The above proposition presents a percentage allocation rule for the mean-variance investor in the market with time-varying Gaussian return. Again, the optimal percentage allocation to the stock has two components: the myopic demand and the intertemporal hedging demand $\hat{H}\left(t, X_{t}\right)$. The policy also inherits all of desirable properties in Basak and Chabakauri [4], as presented in parts (i)-(iii) of Proposition 2, where some properties heavily depend on the magnitude of the market price of risk, therefore we characterize the optimal policy in the average sense. In addition, part (iv) of Proposition 2 shows the monotonicity of optimal mean investment policy in the investment horizon. All implications are similar to those for the stochastic volatility model. Note that the result of monotonicity in the investment horizon does not hold in the model of Basak and Chabakauri [4].

As in the case with stochastic volatility, our model also suggests an asymptotic long-term investment behavior, as $T-t \rightarrow \infty$ in $(28)$ :

$$
\hat{\pi}_{\infty}\left(t, X_{t}\right)=\frac{X_{t}}{\sigma(1+\gamma)}-\frac{\iota \gamma \rho \nu}{(\gamma+1)^{2} \sigma(b+q)}\left(X_{t}+\frac{\lambda}{q} \bar{X}\right)
$$

where $\iota, b$, and $q$ are as given in 29. 
For comparison, we present the optimal investment policy, derived by Kim and Omberg [40] for CRRA utility maximization with risk aversion $\tilde{\gamma}=\gamma+1$, which takes the same form as in 28 except that 29 is replaced by

$$
\iota=1, \quad q=\left[\lambda^{2}+\frac{\gamma}{1+\gamma}\left(\nu^{2}+2 \lambda \rho \nu\right)\right]^{\frac{1}{2}}, \quad b=\lambda+\frac{\gamma \rho \nu}{1+\gamma} .
$$

Table 3 summarizes a comparison of optimal investment policies between our model and Basak-Chabakauri's model under two incomplete market settings: stochastic volatility and Gaussian mean return. Both policies inherit the properties under the complete market. In addition, our stock investment is monotone in investment horizon, while theirs is not.

Table 3 A comparison of optimal policies between our model and Basak-Chabakauri's model under incomplete

\begin{tabular}{ccccc} 
& \multicolumn{5}{c}{ markets $\left(\mu_{t}>r_{t}\right)$} \\
& $\begin{array}{c}\text { wealth-dependent } \\
\text { policy }\end{array}$ & bankruptcy & short sale & $\begin{array}{c}\text { monotonicity of stock } \\
\text { investment in investment horizon }\end{array}$ \\
\cline { 2 - 5 } Basak-Chabakauri & no & possible & never & not always \\
\hline This paper & yes & never & never & yes \\
\hline \multirow{2}{*}{ Basak-Chabakauri } & no & Gaussian Mean Return & not always \\
\cline { 2 - 5 } This paper & yes & never & never & yes \\
\hline
\end{tabular}

\subsection{Numerical Results}

We now conduct numerical analysis to demonstrate our model. Without loss of generality, we focus on the time-varying Gaussian mean-return market, where the parameter values estimated from the historical data by Wachter [55] are used: $\rho=-0.93, r=1.7 \%, \sigma=15 \%$, $X_{0}=\bar{X}=0.273, \lambda=0.27$, and $\nu=6.5 \%$.

\subsubsection{Differences between Our Model and the Mean Variance Model for Terminal}

Wealth. Figure 3 presents the mean dollar amount invested dynamically across the time (or equivalently against different investment horizon cross-sectionally requested by different investors) for different initial wealth. It can be observed that our policy has two monotonic properties: It is an increasing function of both the investment time horizon and the initial 
wealth. This conforms with the empirical finding of Brennan, Schwartz, and Lagnado [14] and Barberis [3] that "the 20-year strategy always invests more in stock than the onemonth strategy, and the differences are often large." As a comparison, Figure 3 also plots the policy of Basak and Chabakauri [4, which is independent of initial wealth and is not monotone against investment horizon.

The analytical investment policy indicates that the myopic term is independent of the investment horizon. Hence, the phenomenon that people having longer investment horizon should invest more in stock arises from the time-dependence of the intertemporal term, known as the hedging demand. From Figure 3 we can observe that as the investment horizon tends to zero, the hedging demand vanishes and the percentage of wealth in stock (i.e. the case of wealth $=1$ ) equals the myopic term $\frac{X_{0}}{(1+\gamma) \sigma}=45.5 \%$.

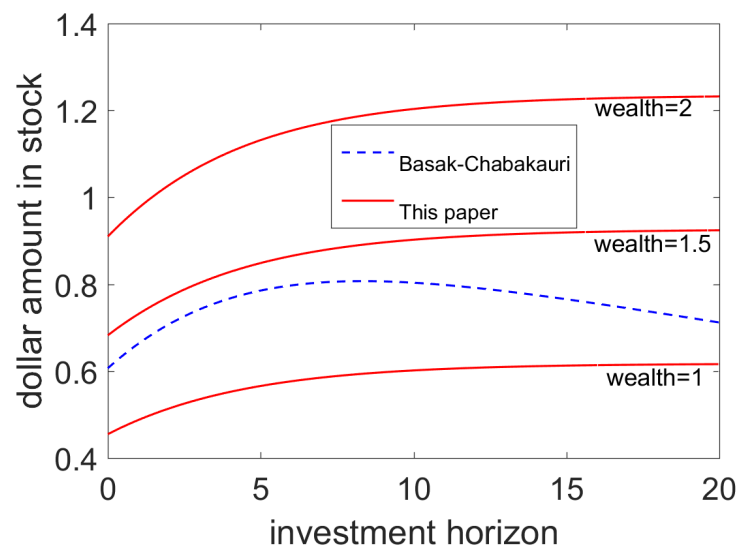

Figure 3 The expected optimal dollar amounts invested in stock with different wealth against investment horizon under the time-varying Gaussian mean return model. Note that curve for wealth $=1$ is also the curve for the relative amount (i.e. the mean percentage allocation) invested in stock. As a comparison, the dash line is from the policy in Basak and Chabakauri [4]. It can be seen that our investment policy satisfies those criteria of the conventional investment wisdom, namely that the richer should invest more dollar amount in stocks, people with longer investment horizon should invest more in stocks, and investors never short a good stock in long run.

Parameter values estimated by Wachter [55] are used: $\rho=-0.93, r=0.017, \sigma=0.15, X_{0}=\bar{X}=0.273, \gamma=3$,

$$
\lambda=0.27 \text {, and } \nu=0.065 \text {. }
$$

Under the time-varying Gaussian mean return market, the stock return may change with $X_{t}$. Figure 4 , reports the expected target annual return of portfolio, $\hat{a}_{0}$, against initial instantaneous stock return rate $\mu_{0}=r+\sigma X_{0}$. It is not surprising to observe that the expected target annual return is increasing with $\mu_{0}$.

As in Campbell and Viceira [17] and Basak and Chabakauri [4], we assess the significance of the intertemporal hedging demands by the ratio of the average intertemporal hedging 


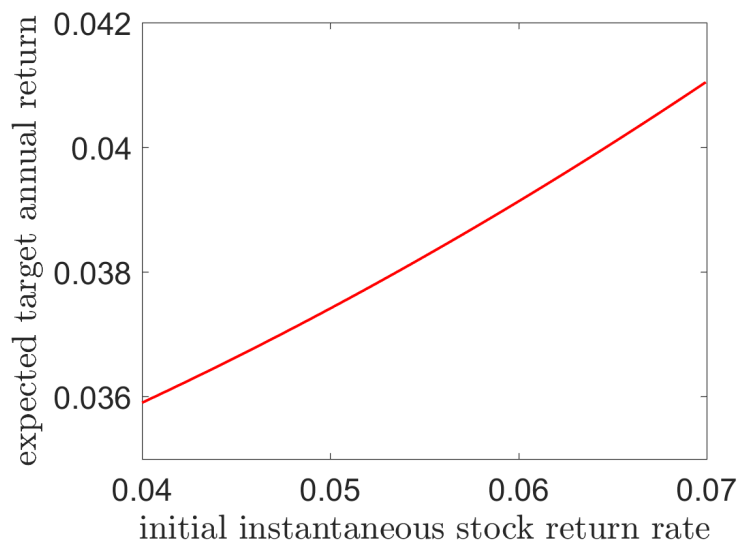

Figure 4 The expected target annual return $\hat{a}_{0}$ against initial instantaneous stock return rate $r+\sigma X_{0}$ under the time-varying Gaussian mean return model. Default parameter values: $\rho=-0.93, r=0.017, \sigma=0.15, T=20$, $\bar{X}=0.273, \gamma=3, \lambda=0.27, \nu=0.065$. It can be seen that the expected target annual return is increasing with the initial instantaneous stock return rate.

demand over the average total demand, namely, $\mathbb{E}\left[\hat{H}\left(t, X_{t}\right)\right] / \mathbb{E}[\hat{\pi}(t, \bar{X})]$. Table 4 reports the ratios with those of Basak and Chabakauri [4] (in parentheses), for different values of $\rho$ and $\lambda$. It can be seen that the ratios are large for longer investment horizons, which indicates that the intertemporal hedging demand is significant for a long-term investment ${ }^{12}$,

\subsubsection{Similarity between Our Mean-Variance Model and the CRRA Model. Using} the default parameter values, we compare in Figure 5 the asset allocation policies derived from the CRRA utility maximization model and our mean-variance model under the Gaussian mean return market setting. It can be seen that the two models yield similar asset allocation policies.

To further examine the linkage between our mean-variance model and the CRRA one, we define the equivalent welfare loss rate, $L$, for an investor with CRRA utility using instead the dynamic mean-variance portfolio as follows:

$$
J_{C R R A}\left(t, X,(1-L) W ; \pi^{C R R A}\right)=J_{C R R A}(t, X, W ; \hat{\pi})
$$

12 Note that the mean-variance preference parameter $\gamma$ does not affect the average intertemporal hedging ratio in Basak and Chabakauri 4] but does in our model, and interestingly, we find that our average hedging ratio converges to the one of Basak and Chabakauri [4] as $\gamma$ in our model tends to infinity (see the last group of Table 4), which is also proved in Online Supplement ??. A possible explanation is that our model is for a relative risk aversion investor, thus the intertemporal hedging ratios depend on the mean-variance preference parameter $\gamma$; as $\gamma$ is sufficiently large, the investor tends to be absolutely risk averse and the intertemporal hedging ratio becomes insensitive to the change of $\gamma$. 
Horizon

\begin{tabular}{|c|c|c|c|c|}
\hline$\rho$ & 6-month & 1-year & 10-year & 20-year \\
\hline-1.00 & $\begin{array}{c}3.90 \\
(5.90)\end{array}$ & $\begin{array}{c}7.18 \\
(10.79)\end{array}$ & $\begin{array}{c}25.98 \\
(38.61)\end{array}$ & $\begin{array}{c}27.88 \\
(41.89)\end{array}$ \\
\hline-0.93 & $\begin{array}{c}3.64 \\
(5.50)\end{array}$ & $\begin{array}{c}6.69 \\
(10.08)\end{array}$ & $\begin{array}{c}24.38 \\
(36.31)\end{array}$ & $\begin{array}{c}26.15 \\
(39.35)\end{array}$ \\
\hline-0.50 & $\begin{array}{c}1.97 \\
(3.00)\end{array}$ & $\begin{array}{l}3.66 \\
(5.54)\end{array}$ & $\begin{array}{c}13.85 \\
(20.86)\end{array}$ & $\begin{array}{l}14.83 \\
(22.46)\end{array}$ \\
\hline 0.00 & $\begin{array}{c}0.00 \\
(0.00)\end{array}$ & $\begin{array}{c}0.00 \\
(0.00)\end{array}$ & $\begin{array}{c}0.00 \\
(0.00)\end{array}$ & $\begin{array}{c}0.00 \\
(0.00)\end{array}$ \\
\hline 0.50 & $\begin{array}{l}-2.02 \\
(-3.09)\end{array}$ & $\begin{array}{l}-3.82 \\
(-5.85)\end{array}$ & $\begin{array}{c}-15.65 \\
(-24.07)\end{array}$ & $\begin{array}{l}-16.64 \\
(-25.45)\end{array}$ \\
\hline 1.00 & $\begin{array}{l}-4.08 \\
(-6.27)\end{array}$ & $\begin{array}{c}-7.79 \\
(-12.03)\end{array}$ & $\begin{array}{c}-33.18 \\
(-51.38)\end{array}$ & $\begin{array}{c}-35.10 \\
(-53.85)\end{array}$ \\
\hline & \multicolumn{4}{|c|}{ Horizon } \\
\hline$\lambda$ & 6 -month & 1-year & 10-year & 20-year \\
\hline 0.00 & $\begin{array}{c}3.88 \\
(5.87)\end{array}$ & $\begin{array}{c}7.59 \\
(11.39)\end{array}$ & $\begin{array}{c}52.16 \\
(70.15)\end{array}$ & $\begin{array}{c}73.23 \\
(91.09)\end{array}$ \\
\hline 0.27 & $\begin{array}{c}3.64 \\
(5.50)\end{array}$ & $\begin{array}{c}6.69 \\
(10.08)\end{array}$ & $\begin{array}{c}24.38 \\
(36.31)\end{array}$ & $\begin{array}{c}26.15 \\
(39.35)\end{array}$ \\
\hline 0.30 & $\begin{array}{c}3.61 \\
(5.46)\end{array}$ & $\begin{array}{c}6.60 \\
(9.94)\end{array}$ & $\begin{array}{c}22.63 \\
(33.83)\end{array}$ & $\begin{array}{c}23.88 \\
(36.03)\end{array}$ \\
\hline 0.60 & $\begin{array}{c}3.36 \\
(5.10)\end{array}$ & $\begin{array}{c}5.78 \\
(8.73)\end{array}$ & $\begin{array}{c}12.56 \\
(19.06)\end{array}$ & $\begin{array}{c}12.60 \\
(19.13)\end{array}$ \\
\hline 0.90 & $\begin{array}{c}3.14 \\
(4.76)\end{array}$ & $\begin{array}{c}5.09 \\
(7.71)\end{array}$ & $\begin{array}{c}8.53 \\
(12.98)\end{array}$ & $\begin{array}{c}8.54 \\
(12.98)\end{array}$ \\
\hline
\end{tabular}

\begin{tabular}{lcccc} 
& \multicolumn{3}{c}{ Horizon } \\
\cline { 2 - 5 }$\gamma$ & 6 -month & 1-year & 10-year & 20-year \\
\hline 1 & 2.09 & 3.87 & 14.37 & 15.33 \\
3 & 3.64 & 6.69 & 24.38 & 26.15 \\
5 & 4.22 & 7.76 & 28.14 & 30.27 \\
10 & 4.79 & 8.78 & 31.74 & 34.25 \\
30 & 5.24 & 9.61 & 34.66 & 37.5 \\
60 & 5.37 & 9.84 & 35.46 & 38.41 \\
& $(5.50)$ & $(10.08)$ & $(36.31)$ & $(39.35)$ \\
\hline
\end{tabular}

Table 4 Average intertemporal hedge ratios in percentage. The default parameters are $\gamma=3$ and those in Wachter [55] as used throughout this section. The numbers in parentheses are taken from Basak and Chabakauri [4]. It appears that the intertemporal term is quite significant especially for a long time horizon.

where the left and right hand sides are the expected CRRA utilities from the optimal CRRA policy $\pi^{C R R A}$ with risk aversion $\tilde{\gamma}$ and from our optimal mean-variance policy $\hat{\pi}$ with mean-variance preference $\gamma=\tilde{\gamma}-1$, respectively. Due to the homogeneity, it is easy to see that $L$ does not depend on initial wealth $W$. Figure 6 plots the certainty equivalent welfare loss rate $L$ for a CRRA investor using the dynamic mean variance strategy against 


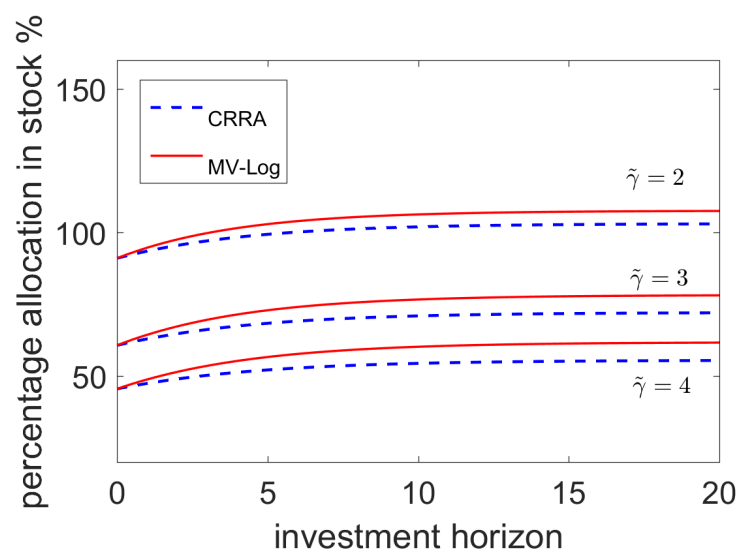

Figure 5 The optimal mean percentage allocations in stock against investment horizon for the CRRA utility maximization model with risk aversion $\tilde{\gamma}$ and our mean-variance model with mean-variance preference $\gamma=\tilde{\gamma}-1$ under a Gaussian mean return market setting. Parameter values estimated by Wachter [55] are used: $\rho=-0.93$, $r=0.017, \sigma=0.15, X_{0}=\bar{X}=0.273, \lambda=0.27$, and $\nu=0.065$.

investment horizon for different risk aversion. Note that $L$ is small; in particular, with 20 years investment horizon, the largest loss rate is no more than $1.2 \%$. Furthermore, the lower the risk aversion parameter, the lower the equivalent welfare loss rate, because our meanvariance model is reduced to the log utility maximization model when we send $\tilde{\gamma} \rightarrow 1.13$ On the other side, it is not surprising that the welfare loss is increasing with investment horizon.

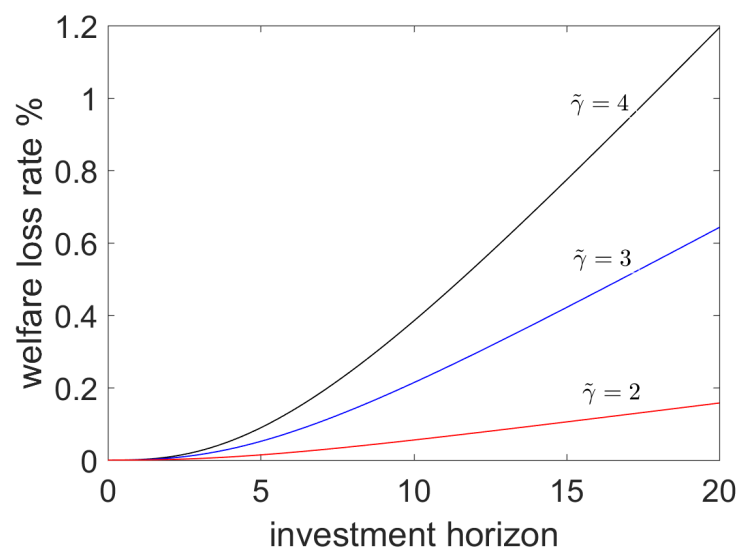

Figure 6 The certainty equivalent welfare loss rate when an investor having a CRRA utility instead uses our mean-variance policy under a Gaussian mean return market setting. Note that the loss rate is small, indicating the similarity between the CRRR optimal policy and our dynamic mean variance policy. Parameter estimated in Wachter [55] are used: $\rho=-0.93, r=0.017, \sigma=0.15, X_{0}=\bar{X}=0.273, \lambda=0.27$, and $\nu=0.065$.

\footnotetext{
${ }^{13}$ It should be pointed out that this monotonicity may not be true for a extremely big $\tilde{\gamma}$ for which there is almost no investment in stock and few welfare losses would be incurred as a result.
} 


\section{Extension to Portfolio Constraints}

We shall show that our analysis can be extended to incorporate portfolio constraints. The extension to multiple stocks is placed in Online Supplement ??.

We impose the following portfolio constraints to the incomplete market setting described in Section 4

$$
\underline{\pi}_{t} \leq \pi_{t} \leq \bar{\pi}_{t} \text { for any } t \in[0, T]
$$

where $\underline{\pi}_{t}$ and $\bar{\pi}_{t}$ are both adapted stochastic processes, and $-\infty \leq \underline{\pi}_{t}<\bar{\pi}_{t} \leq+\infty$. The constraints cover the widely used no-short-sale constraint $\left(\underline{\pi}_{t} \equiv 0\right)$ and no-borrowing constraint $\left(\bar{\pi}_{t} \equiv 1\right)$. We can similarly define equilibrium solutions under this setting. It turns out that a semi-analytical equilibrium solution is still available as follows.

Proposition 3. Consider the mean-variance criterion (7) subject to (6) in the incomplete market with portfolio constraints (32). Assume

$$
\mathbb{E}\left[\int_{0}^{T} \theta_{s}^{2} d s\right]<+\infty, \quad \mathbb{E}\left[e^{-\frac{\gamma}{1+\gamma}\left(\rho \int_{0}^{T}\left(r_{t}+\theta_{t}^{2} / 2\right) d t+\int_{0}^{T} \theta_{t} d B_{t}^{X}\right)}\right]<+\infty .
$$

Then we have the following statements:

(i) There is an equilibrium policy

$$
\hat{\pi}_{t}=\left[\left(\frac{\mu_{t}-r_{t}}{(1+\gamma) \sigma_{t}^{2}}-\frac{\rho \gamma Z_{t}}{(1+\gamma) \sigma_{t}}\right) \vee \underline{\pi}_{t}\right] \wedge \bar{\pi}_{t}
$$

where $Z_{t}$ is uniquely determined by the BSDE (15) with

$$
g(t, z)=r_{t}+\frac{1}{2} \theta_{t}^{2}-\frac{1}{2}\left(\left(\theta_{t}-\sigma_{t} \underline{\pi}_{t}\right) \wedge \frac{\gamma\left(\theta_{t}+\rho z\right)}{1+\gamma} \vee\left(\theta_{t}-\sigma_{t} \bar{\pi}_{t}\right)\right)^{2}
$$

(ii) If $\underline{\pi}_{t}=\underline{\pi}\left(t, X_{t}\right)$ and $\bar{\pi}_{t}=\bar{\pi}\left(t, X_{t}\right)$ for some deterministic functions $\underline{\pi}(\cdot, \cdot)$ and $\bar{\pi}(\cdot, \cdot)$, there exists a deterministic function $f(\cdot, \cdot)$ such that the solution $(Y, Z)$ for the BSDE (15)

\footnotetext{
${ }^{14}$ Here we use the notations $a \vee b=\max (a, b)$ and $a \wedge b=\min (a, b)$. In addition, to ensure the equilibrium policy $\hat{\pi}$ depends on $X_{t}$ and $t$ only, we assume that $\underline{\pi}$ and $\bar{\pi}$ are $\mathcal{F}_{t}^{B^{X}}$-adapted.
} 
with (34) satisfies $Y_{t}=f\left(t, X_{t}\right)$ and $Z_{t}=\nu(t, x) \frac{\partial f}{\partial x}$. Moreover, if $f(\cdot, \cdot)$ is $C^{1,2}$, then $f(\cdot, \cdot)$ solves the PDE problem (18) with $g(\cdot, \cdot)$ as given by (34).

In a complete market with constant parameters, the optimal policy (33) reduces to

$$
\hat{\pi}_{t}:=\left(\frac{\mu-r}{(1+\gamma) \sigma^{2}} \vee \underline{\pi}_{t}\right) \wedge \bar{\pi}_{t}
$$

which is the same as the policy under the CRRA utility maximization with relative risk aversion parameter $\tilde{\gamma}=\gamma+1$ (see, e.g., Cvitanic and Karazatzs [23] and Dai, Jin, and Liu [25]). The policy is myopic with respect to portfolio constraints in the sense that no action is taken before the constraints are binding.

However, in an incomplete market, the optimal policy is usually non-myopic with respect to portfolio constraints because the hedging demand is affected by the constraints through $g(\cdot, \cdot)$. Figure 7 shows the stock allocation for the stochastic volatility model with and without portfolio constraints, where the default parameter values are $\alpha=1, \rho=-0.52$, $r=0.017, \delta=1, X_{0}=\bar{X}=0.04, \gamma=2.5, \lambda=0.3374, T=15, \bar{\nu}=0.6503, \underline{b}_{t} \equiv 0.4$, and $\bar{b}_{t}=+\infty$. For this special case without portfolio constraint, our analytical result shows that the percentage stock allocation is independent of the level of market price of risk. The same property holds for the portfolio constraint case. Moreover, we can observe a nonmyopic phenomenon: In anticipation of the constraint being likely binding in the future, the investor reacts before binding. Since the constraint implies a minimum fraction of wealth invested in stock and the correlation $\rho$ is negative, more hedging demand is incurred such that the investor raises stock investment before binding.

\section{Conclusion}

We propose a dynamic, time consistent, mean-variance model for portfolio log-returns. The optimal policy is obtained analytically even in some incomplete markets. We show that the asset allocation policy satisfies three investment criteria from the conventional wisdom, such that (1) rich people should invest more dollar amount in risky assets; (2) the longer the investment horizon, the more proportional amount of money should be invested in risky assets; (3) for long-term investment, people should not short sell major stock indices, whose returns are higher than the risk-free rate. These three requirements pose challenges to existing portfolio theories. The model can also elicit the mean-variance 


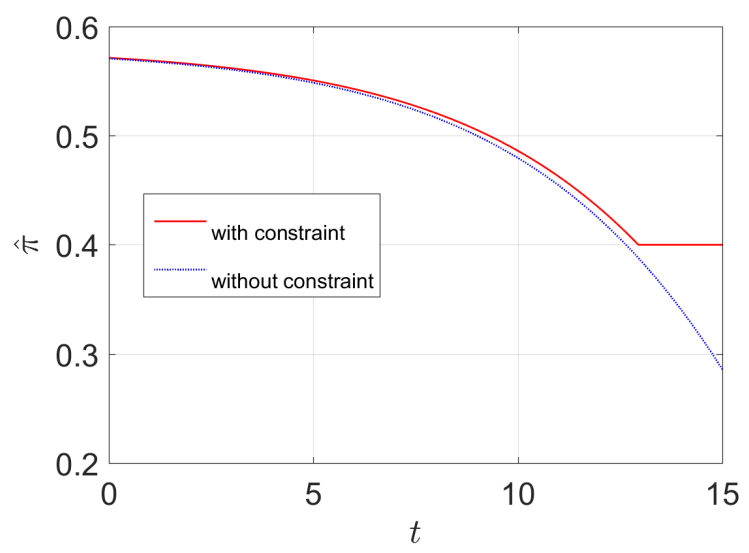

Figure 7 The fraction of total wealth in stock against time for the stochastic volatility model with and without portfolio constraints. Default parameter values: $\alpha=1, \rho=-0.52, r=0.017, \delta=1, X_{0}=\bar{X}=0.04, \gamma=2.5$,

$$
\lambda=0.3374, T=15, \bar{\nu}=0.6503, \underline{b}_{t} \equiv 0.4 \text {, and } \bar{b}_{t}=+\infty \text {. }
$$

preference parameter by asking investors to input their desired expected returns in a fictitious complete market. Further, the model is extended to incorporate portfolio constraints and to handle multiple stocks.

It will be interesting to investigate further how to apply the model to study the asset allocation part of robo-advising, by incorporating other factors such as taxes, retirement and educational needs of investors, and age of investors. We will leave this for future study.

\section{Acknowledgements}

We are grateful to participants at The 3rd Berlin-Princeton-Singapore Workshop on Quantitative Finance, The 5th AQFC, 2018 Informs Annual Meeting, and The 12th Annual Risk Management Conference for useful discussions. Dai is supported by Singapore MOE AcRF grants R-146-000-243-114 and R-703-000-032-112, and NSFC grant 11671292. Xu is supported by NSFC grant 11871050 .

\section{References}

[1] Ait-Sahaly, Y., and Brandt, M. W. (2001). Variable selection for portfolio choice. The Journal of Finance, 56, 1297-1351.

[2] Bajeux-Besnainou, I., and Portait, R. (1998). Dynamic asset allocation in a mean-variance framework. Management Science, 44(11), 79-95.

[3] Barberis, N. (2000). Investing for the long run when returns are predictable. The Journal of Finance, 55, $225-264$.

[4] Basak, S., and Chabakauri, G. (2010). Dynamic mean-variance asset allocation. Review of Financial Studies, 23, 2970-3016.

[5] Basak, S., and Chabakauri, G. (2012). Dynamic hedging in incomplete markets: A simple solution. Review of Financial Studies, 25, 1845-1896. 
[6] Basak, S., and Makarov, D. (2014). Strategic asset allocation in money management. The Journal of Finance, $69,179-217$.

[7] Bielecki, T., Jin, H., Pliska, S. R., and Zhou, X. Y. (2005). Continuous-time mean-variance portfolio selection with bankruptcy prohibition. Mathematical Finance, 15, 213-44.

[8] Bielecki, T., and Pliska, H. (1999). Risk-sensitive dynamic asset management. Applied Mathematics and Optimization 39, 337-360.

[9] Björk, and Murgoc, A. (2014). A theory of Markovian time-inconsistent stochastic control in discrete time. Finance and Stochastics, 18, 545-592.

[10] Björk, T., Khapko, M., and Murgoc, A. (2017). On time-inconsistent stochastic control in continuous time. Finance and Stochastics, 21, 331-360.

[11] Björk, T., Murgoci, A., and Zhou, X. Y. (2014). Mean-variance portfolio optimization with state-dependent risk aversion. Mathematical Finance, 24, 1-24.

[12] Bouchard, B. and Touzi, N. (2004). Discrete-time approximation and Monte-Carlo simulation of backward stochastic differential equations. Stochastic Process and Application, 111 175-206.

[13] Brandt, M. W. (1999). Estimating portfolio and consumption choice: A conditional Euler equations approach. The Journal of Finance, 54, 1609-1645.

[14] Brennan, M. J., Schwartz, E. S., and Lagnado, R. (1997). Strategic asset allocation, Journal of Economic Dynamics and Control, 21, 1377-1403.

[15] Briand, P., and Hu, Y. (2006). BSDE with quadratic growth and unbounded terminal value. Probability Theory and Related Fields, 136(4), 604-618.

[16] Brown, K. C., Harlow, W. V., and Starks, L. T. (1996). Of tournaments and temptations: An analysis of managerial incentives in the mutual fund industry. The Journal of Finance, 51, 85-110.

[17] Campbell, J. Y., and Viceira, L. M. (1999). Consumption and portfolio decisions when expected returns are time varying. Quarterly Journal of Economics, 114, 433-495.

[18] Campbell, J. Y., and Viceira, L. M. (2002). Strategic asset allocation: portfolio choice for long-term investors. Clarendon Lectures in Economic.

[19] Chacko, G., and Viceira, L. M. (2005). Dynamic consumption and portfolio choice with stochastic volatility in incomplete markets. Review of Financial Studies, 18, 1369-402.

[20] Chassagneux, J.F. and Richou, A. (2016). Numerical simulation of quadratic BSDEs. The Annals of Applied Probability, 26, 263-304.

[21] Chevalier, J., and Ellison, G. (1997). Risk taking by mutual funds as a response to incentives. Journal of Political Economy, 105, 1167-1200.

[22] Cochrane, J. (2014). A mean-variance benchmark for intertemporal portfolio theory. Journal of Finance, 69, 1-49.

[23] Cvitanic, J., and Karatzas, I. (1992). Convex duality in constrained portfolio optimization. Ann. Appl. Probability, 2, 767-818.

[24] Cvitanic, J., Lazrak, A., and Wang, T. (2008). Implications of the Sharpe ratio as a performance measure in multi-period settings. Journal of Economic Dynamics and Control, 32, 1622-49.

[25] Dai, M., Jin, H. Q., and Liu, H. (2011). Illiquidity, position limits, and optimal investment for mutual funds. Journal of Economic Theory 146, 1598-1630. 
[26] Dai, M., Xu, Z. Q., and Zhou, X. Y. (2010). Continuous-time mean-variance portfolio selection with proportional transaction costs. SIAM Journal on Financial Mathematics 1, 96-125.

[27] Davis, M., and Lleo, S. (2014). Risk-sensitive investment Management. World Scientific Publishing Company.

[28] Ekeland, I., and Lazrak, A. (2006). Being serious about non-commitment: subgame perfect equilibrium in continuous time. arXiv preprint math/0604264.

[29] Ekeland, I., and Pirvu, T. A. (2008). Investment and consumption without commitment. Mathematics and Financial Economics, 2, 57-86.

[30] Fan, J., Fan, Y. and Lv, J. (2008). High dimensional covariance matrix estimation using a factor model. Journal of Econometrics, 147, 186-197.

[31] Fan, J., Liao, Y. and Liu, H. (2016). An overview on the estimation of large covariance and precision matrices. Econometrics Journal, 19, C1-C32.

[32] Fan, J., Liao, Y. and Micheva, M. (2013). Large Covariance Estimation by Thresholding Principal Orthogonal Complements (with discussion). Journal of Royal Statistical Society B, 75, 603-680.

[33] Fan, J., Zhang, J., and Yu, K. (2012). Vast portfolio selection with gross-exposure constraints. Journal of American Statistical Association, 107, 592-606.

[34] Graf, S. and Luschgy, H. (2000). Foundations of Quantization for Probability Distributions. Lecture Notes in Math. 1730. Springer, Berlin.

[35] Gruber, M. J. (1996). Another puzzle: The growth in actively managed mutual funds. The Journal of Finance, $51,783-810$.

[36] He, X. and Jiang, Z. (2018). Dynamic mean-risk asset allocation and myopic strategies: A universal portfolio rule. Working paper, Chinese University of Hong Kong.

[37] Heston, S. L. (1993). A closed-form solution for options with stochastic volatility with applications to bond and currency options. Review of Financial Studies, 6, 327-43.

[38] Jagannathan, R., and Ma, T. (2003). Risk Reduction in Large Portfolios: Why Imposing the Wrong Constraints Helps. Journal of Finance, 58, 165184.

[39] Jorion, P. (1986). Bayes-Stein Estimation for Portfolio Analysis. Journal of Financial and Quantitative Analysis,, 21, 279-292.

[40] Kim, T. S., and Omberg, E. (1996). Dynamic nonmyopic portfolio behavior. Review of Financial Studies, 9, $141-61$.

[41] Kydland, F. E., and Prescott, E. C. (1982). Time to build and aggregate fluctuations. Econometrica, 50, 13451370.

[42] Li, D., and Ng, W. L. (2000). Optimal Dynamic portfolio selection: multiperiod mean-variance formulation. Mathematical Finance, 10, 387-406.

[43] Liu, J. (2001). Dynamic portfolio choice and risk aversion. Working Paper, UCLA, SSRN-id287095.

[44] Liu, J. (2007). Portfolio selection in stochastic environments. Review of Financial Studies, 20, 1-39.

[45] Luenberger, D. G. (1993). A preference foundation for log mean-variance criteria in portfolio choice problems. Journal of Economic Dynamics and Control, 17, 887-906.

[46] Mankiw, N. G., and Zeldes, S. P. (1991). The consumption of stockholders and nonstockholders. Journal of Financial Economics, 29, 97-112.

[47] Markowitz, H. (1952). Portfolio selection. Journal of Finance, 7, 77-91. 
[48] Mehra, R., and Prescott, E. C. (1985). The equity premium: A puzzle. Journal of Monetary Economics, 15, 145-161.

[49] Merton, R. C. (1971). Optimum consumption and portfolio rules in a continuous-time model. Journal of Economic Theory, 3, 373-413.

[50] Pages, G., Pham, H. and Printems, J. (2004). Optimal quantization methods and applications to numerical problems in finance. In Handbook of Computational and Numerical Methods in Finance 253-297. Birkhauser, Boston, MA.

[51] Peleg, B., and Yaari, M. E. (1973). On the existence of consistent course of action when tastes are changing. Review of Economic Studies, 40, 391-401.

[52] Shapiro, A. (2012). Time consistency of dynamic risk measures. Operations Research Letters, 40, $436-439$.

[53] Sirri, E. R., and Tufano, P. (1998). Costly search and mutual fund flows. The Journal of Finance, 53, $1589-1622$.

[54] Strotz, R. H. (1956). Myopia and inconsistency in dynamic utility maximization. Review of Economic Studies, $23,165-80$.

[55] Wachter, J. A. (2002). Portfolio and consumption decisions under mean-reverting returns: An explicit solution for complete market. Journal of Financial and Quantitative Analysis, 37, 63-91.

[56] Zhang, J. (2004). A numerical scheme for BSDEs. Annals of Applied Probability, 14, 459-488.

[57] Zhou, X. Y., and Li, D. (2000). Continuous-time mean-variance portfolio selection: A stochastic LQ framework. Applied Mathematics and Optimization, 42, 19-53. 


\section{Online Supplement}

\section{A Dynamic Mean-Variance Analysis for Log Returns}

Min Dai, Hanqing Jin, Steven Kou, Yuhong Xu

\section{A. Extension to Multiple Stocks}

We now extend our baseline analysis to the case of multiple stocks. Assume that a riskless bond and $N$ risky stocks are available for investment. The stock prices $S_{i}, i=1, \ldots, N$, satisfy

$$
\frac{d S_{i t}}{S_{i t}}=\mu_{i}\left(t, X_{t}\right) d t+\sum_{j=1}^{N} \sigma_{i j}\left(t, X_{t}\right) d B_{j t},
$$

where $B_{t} \equiv\left(B_{1 t}, \ldots, B_{N t}\right)^{\top}$ is a standard $N$-dimensional Brownian motion, and $\mu_{i}$ and $\sigma_{i j}$ are deterministic functions of $t$ and $K$ state variables, $X=\left(X_{1}, \ldots, X_{K}\right)^{\top}$, which satisfy

$$
d X_{j t}=m_{j}\left(t, X_{t}\right) d t+\sum_{k=1}^{K} \nu_{j k}\left(t, X_{t}\right) d B_{k t}^{X},
$$

where $B_{t}^{X} \equiv\left(B_{1 t}^{X}, \ldots, B_{K t}^{X}\right)^{\top}$ is another standard $K$-dimensional Brownian motion, and $m_{j}$ and $\nu_{j k}$ are deterministic functions of $t$ and $X$. We assume $E\left(B_{i t} B_{j t}^{X}\right)=\rho_{i j} d t$, for all $i$ and $j$.

To simplify notations, we denote by $\mu \equiv\left(\mu_{1}, \ldots, \mu_{N}\right)^{\top}$ the vector of stock expected return rates, and $\sigma \equiv\left(\sigma_{i j}\right)_{N \times N}$ the volatility matrix (assumed invertible), respectively. Similarly, $m \equiv\left(m_{1}, \ldots, m_{K}\right)^{\top}$ and $\nu \equiv\left(\nu_{i j}\right)_{K \times K}$ denote the expected growth and the volatility matrix of $X$, respectively, and $\rho=\left(\rho_{i j}\right)_{N \times K}$ denotes the correlation matrix of $B$ and $B^{X}$. The investor's wealth is then governed by

$$
\frac{d W_{t}}{W_{t}}=\left(r+\pi_{t}^{\top}(\mu-r \mathbf{1})\right) d t+\pi_{t}^{\top} \sigma d B_{t},
$$

where $\pi=\left(\pi_{1}, \ldots, \pi_{N}\right)^{\top}$ denotes the vector of percentage allocations of wealth in the $N$ stocks, and 1 denotes the $N$-dimension unit vector $(1, \ldots, 1)^{\top}$. Consequently, the log-return $R_{T}=\ln W_{T}$ follows

$$
d R_{t}=\left[r+\pi_{t}^{\top}(\mu-r \mathbf{1})-\frac{1}{2} \pi_{t}^{\top} \sigma \sigma^{\top} \pi_{t}\right] d t+\pi_{t}^{\top} \sigma d B_{t} .
$$

The dynamic mean-variance problem is the same as before. We maximize the objective function (7) subject to (36). By analogous analysis, we have the following result. 
Proposition 4. Assume the above multiple-stock market setting with $\mathbb{E}\left[\int_{0}^{T} \theta_{s}^{2} d s\right]<+\infty$ and $\mathbb{E}\left[e^{\frac{\gamma^{2}}{(1+\gamma)^{2}}\left(\int_{0}^{T}\left[|\rho|^{2}\left(r_{t}+\frac{1+2 \gamma}{(1+\gamma)^{2}} \frac{\left|\theta_{t}\right|^{2}}{2}\right)-\frac{1}{2} \frac{\gamma^{2}}{(1+\gamma)^{2}}\left|\rho^{\top} \theta_{t}\right|^{2}\right] d t-\int_{0}^{T} \theta_{t}^{\top} \rho d B_{t}^{X}\right)}\right]<+\infty$.

(i) There is an equilibrium policy

$$
\hat{\pi}\left(t, X_{t}\right)=\frac{1}{1+\gamma}\left(\sigma \sigma^{\top}\right)^{-1}(\mu-r \mathbf{1})-\frac{\gamma}{1+\gamma}\left(\sigma^{-1}\right)^{\top} \rho Z_{t}
$$

where $Z_{t}$ is uniquely determined by the BSDE with multiple Brownian motions

$$
d Y_{t}=-g\left(t, Z_{t}\right) d t+Z_{t}^{\top} d B_{t}^{X}, \quad Y_{T}=0
$$

with $g(t, z)=r_{t}+\frac{1}{2}\left|\theta_{t}\right|^{2}-\frac{1}{2} \frac{\gamma^{2}}{(1+\gamma)^{2}}\left|\theta_{t}+\rho z\right|^{2}$.

(ii) In the case $K=1$, i.e., $X$ is a 1-dimensional process generated by a 1-dimensional standard Brownian motion $B_{t}^{X}$, denote 15

$$
\begin{aligned}
& k=\frac{\gamma}{1+\gamma}, \quad \beta(t, x)=\frac{\theta(t, x)^{\top} \rho}{|\rho|^{2}} . \\
& \alpha(t, x)=r(t, x)+\frac{1-k^{2}}{2}|\theta(t, x)|^{2}+\frac{k^{2}}{2} \frac{\left|\theta(t, x)^{\top} \rho\right|^{2}}{|\rho|^{2}} .
\end{aligned}
$$

Then

$$
Y_{t}=\frac{-1}{|k \rho|^{2}} \ln \mathbb{E}_{t}\left[e^{-|k \rho|^{2}\left(\int_{t}^{T} \alpha\left(s, X_{s}\right) d s+\int_{t}^{T} \beta\left(s, X_{s}\right) d B_{s}^{X}\right)}\right]
$$

(iii) There exists a deterministic function $f(\cdot, \cdot)$ such that the solution $(Y, Z)$ for the BSDE (38) satisfies $Y_{t}=f\left(t, X_{t}\right)$ and $Z_{t}=\nu(t, X) \frac{\partial f}{\partial X}$. Moreover, if $f(\cdot, \cdot)$ is $C^{1,2}$, then $f=f(t, X)$ satisfies the following PDE:16

$$
\begin{aligned}
\frac{\partial f}{\partial t} & +\left(m-\frac{\gamma^{2}}{(1+\gamma)^{2}} \nu \rho^{\top} \sigma^{-1}(\mu-r \mathbf{1})\right)^{\top} \frac{\partial f}{\partial X}+\frac{1}{2} \operatorname{Tr}\left(\nu^{\top} \frac{\partial^{2} \mathrm{f}}{\partial \mathrm{X}^{2}} \nu\right) \\
& -\frac{1}{2} \frac{\gamma^{2}}{(1+\gamma)^{2}}\left(\rho \nu^{\top} \frac{\partial f}{\partial X}\right)^{\top} \rho \nu^{\top} \frac{\partial f}{\partial X}+\frac{2 \gamma+1}{2(\gamma+1)^{2}}(\mu-r \mathbf{1})^{\top}\left(\sigma \sigma^{\top}\right)^{-1}(\mu-r \mathbf{1})+r=0,
\end{aligned}
$$

${ }^{15}$ Again, we consider the non-trivial case $|\rho| \neq 0$.

${ }^{16}$ Here $\operatorname{Tr}\left(\nu^{\top} \frac{\partial^{2} f}{\partial X^{2}} \nu\right)$ means the trace of the matrix $\nu^{\top} \frac{\partial^{2} f}{\partial X^{2}} \nu$. 
subject to terminal condition $f(T, X)=0$, and we denote

$$
\frac{\partial f}{\partial X} \equiv\left(\frac{\partial f}{\partial X_{1}}, \ldots, \frac{\partial f}{\partial X_{K}}\right)^{\top} \quad \text { and } \quad \frac{\partial^{2} f}{\partial X^{2}} \equiv\left(\frac{\partial^{2} f}{\partial X_{i} \partial X_{j}}\right)_{K \times K}
$$

respectively.

In the case that $X$ is a multi-dimensional process generated by a multi-dimensional Brownian motion, due to the interplay among the components of Brownian motion, we cannot get an explicit solution for the BSDE (38) in terms of conditional expectation as done in Basak and Chabakauri [4]. Note that a general quadratic BSDE usually does not have an explicit solution except for the 1-dimension case. For example, under some mild regularity condition, the quadratic BSDE

$$
d Y_{t}=-\frac{1}{2} \alpha Z_{t}^{2} d t+Z_{t} d B_{t}, Y_{T}=\xi
$$

with 1-dimension standard Brownian motion $B_{t}$ admits an explicit solution $Y_{t}=\frac{1}{\alpha} \ln \mathbb{E}_{t}\left[e^{\alpha \xi}\right]$; while in the case of 2 -dimension standard Brownian motion $\left(B_{1, t}, B_{2, t}\right)$, the quadratic BSDE

$$
d Y_{t}=-\frac{1}{2}\left(\alpha Z_{1, t}^{2}+\beta Z_{2, t}^{2}\right) d t+Z_{1, t} d B_{1, t}+Z_{2, t} d B_{2, t}, Y_{T}=\xi
$$

does not admit an explicit solution unless $\alpha=\beta$.

However, we can still use Monte-Carlo simulations for BSDE (38) to numerically find the equilibrium policy for the high-dimensional problem.17 See Online Supplement B.

It can be seen that the optimal percentage allocation still contains two parts: one reflects the myopic demand, and the other reflects the intertemporal hedging demand. As in Basak and Chabakauri [4], Proposition 4 reveals a cross-correlation effect on the optimal allocation. Due to this cross-correlation effect, some conventional investment rules that hold in the single-stock case may no longer be true. For example, given all positive stock premiums (i.e., $\mu_{i}>r$ for all $i$ ), short-selling some stocks is likely optimal. 18 Hence, for the multiple stocks case, it may make more sense to examine the properties of the total percentage of wealth invested in stocks, $\mathbf{1}^{\top} \hat{\pi}$.

\footnotetext{
17 This comment also applies to the portfolio constraints case in Proposition 3.

${ }^{18}$ For example, given $\mu_{i}>\mu_{j}>r$, we may long stock $i$ and short stock $j$ in some scenarios.
} 


\section{The overall no-short-sale}

Let us first examine when $\mathbf{1}^{\top} \hat{\pi} \geq 0$, which implies the overall no-short-sale. Construct a covariance-weighted portfolio $\tilde{\pi}=\left(\sigma \sigma^{\top}\right)^{-1} \mathbf{1}$, whose wealth process is denoted by $W_{t}^{\tilde{\pi}}$. Let $W_{t}^{\hat{\pi}}$ be the wealth process associated with the optimal portfolio $\hat{\pi}$. It is easy to see

$$
\mathbb{E}_{t}\left[d W_{t}^{\tilde{\pi}} d W_{t}^{\hat{\pi}}\right]=\tilde{\pi}^{\top} \sigma \sigma^{\top} \hat{\pi} W^{\tilde{\pi}} W^{\hat{\pi}} d t=\mathbf{1}^{\top} \hat{\pi} W^{\tilde{\pi}} W^{\hat{\pi}} d t
$$

We then infer that $\mathbf{1}^{\top} \hat{\pi} \geq 0$ if and only if the returns of the covariance-weighted portfolio and the optimal portfolio are not negatively correlated.

\section{The monotonicity of the total percentage stock allocation with investment horizon}

Now let us examine when the total percentage stock allocation is monotonically increasing with investment horizon, namely, the monotonicity of $\mathbf{1}^{\top} \hat{\pi}$ with respect to $T-t$. For simplicity, we assume $K=1$, and $\mu=\mu(X), r=r(X), \sigma=\sigma(X), m=m(X), \nu=\nu(X)>0$, and $\rho=\rho(X)$ are all independent of time. It follows

$$
\frac{\partial}{\partial t}\left(\mathbf{1}^{\top} \hat{\pi}\right)=-\frac{\gamma}{1+\gamma} \nu\left(\rho^{\top} \sigma^{-1} \mathbf{1}\right) \frac{\partial}{\partial t}\left(\frac{\partial f}{\partial X}\right)
$$

Assuming $\frac{\partial}{\partial t}\left(\frac{\partial f}{\partial X}\right)<0,19$ we deduce that the total percentage stock allocation is monotonically increasing with investment horizon $T-t$ if and only if

$$
\rho^{\top} \sigma^{-1} \mathbf{1}<0
$$

This condition reduces to $\rho<0$ for $N=1$, consistent with the result obtained in Section 4 . Note that the covariance

$$
\mathbb{E}_{t}\left[d W_{t}^{\tilde{\pi}} d X_{t}\right]=\rho^{\top} \sigma^{-1} \mathbf{1} \nu W_{t}^{\tilde{\pi}} d t
$$

where $W_{t}^{\tilde{\pi}}$ is the wealth associated with the covariance-weighted portfolio $\tilde{\pi}$ as defined before. Hence, the condition (39) implies a negative correlation between $d X_{t}$ and the return of the covariance-weighted portfolio.

\footnotetext{
${ }^{19}$ As in the single-stock case, we will see that this is true for the time-varying Gaussian mean return model with two stocks presented in Online Supplement C.8.
} 


\section{A time-varying Gaussian mean returns model with two stocks}

To better understand (39), we provide a time-varying Gaussian mean returns model with two stocks. More specifically, the two stock prices have dynamics

$$
\frac{d S_{i t}}{S_{i t}}=\left(r+\delta_{i} X_{t}\right) d t+\sigma_{i} d \hat{B}_{i t}, \quad i=1,2,
$$

where $r, \delta_{1}, \delta_{2}, \sigma_{1}, \sigma_{2}$ are all positive constants, $\hat{B}_{1 t}$ and $\hat{B}_{2 t}$ are two one-dimension Brownian motions with constant correlation $\rho_{S}$. The state $X_{t}$ follows the mean-reverting process (27). We assume $E\left(B_{t}^{X} \hat{B}_{i t}\right)=\rho_{i}^{S X} d t, i=1,2$.

In Online Supplement C.8, we show that an analytical formula of $f(t, X)$ is available, $\frac{\partial}{\partial t}\left(\frac{\partial f}{\partial X}\right)<0$, and

$$
\rho^{\top} \sigma^{-1}=\frac{1}{\sigma_{1} \sigma_{2}\left(1-\rho_{S}^{2}\right)}\left(\sigma_{2}\left(\rho_{1}^{S X}-\rho_{S} \rho_{2}^{S X}\right), \sigma_{1}\left(\rho_{2}^{S X}-\rho_{S} \rho_{1}^{S X}\right)\right) .
$$

As a consequence, the condition (39) is equivalent to

$$
\sigma_{2}\left(\rho_{1}^{S X}-\rho_{S} \rho_{2}^{S X}\right)+\sigma_{1}\left(\rho_{2}^{S X}-\rho_{S} \rho_{1}^{S X}\right)<0 .
$$

When $\rho_{1}^{S X}<0, \rho_{2}^{S X}<0$ and $\rho_{S}<0$, (41) apparently holds, then we deduce that $\pi_{1}+\pi_{2}$ is increasing with investment horizon. In fact, from (37) and (40) we can infer that both $\pi_{1}$ and $\pi_{2}$ are increasing with investment horizon given the negative correlations.

\section{Two numerical examples}

Now we present numerical results for the time-varying Gaussian mean-return model with two stocks. Consider the first case in which $\rho_{1}^{S X}=-0.15, \rho_{2}^{S X}=-0.93, \rho_{S}=-0.1, \sigma_{1}=0.15$, $\sigma_{2}=0.17, \delta_{1}=0.09, \delta_{2}=0.11$, and other parameters are the same as in Figure 4 . It can be seen that the returns of two stocks are both negatively correlated with the market state $X_{t}$, and the two stock returns are negatively correlated. Hence, from (37) and (40), we infer that the percentage allocation in each stock must be monotonically increasing with investment horizon, which is demonstrated by Figure 8 .

Consider the second case in which the two stock returns are positively correlated $\left(\rho_{S}=0.3\right)$, and other parameters are the same as in the first case. It is easy to verify $\sigma_{2}\left(\rho_{1}^{S X}-\rho_{S} \rho_{2}^{S X}\right)=$ 0.0119 and $\sigma_{1}\left(\rho_{2}^{S X}-\rho_{S} \rho_{1}^{S X}\right)=-0.0272$. According to our analysis, the percentage allocation in stock 1 is decreasing with investment horizon, while the percentage allocation in stock 2 is increasing with investment horizon. Note that $\sigma_{2}\left(\rho_{1}^{S X}-\rho_{S} \rho_{2}^{S X}\right)+\sigma_{1}\left(\rho_{2}^{S X}-\rho_{S} \rho_{1}^{S X}\right)=$ $0.0119-0.0272<0$. As a result, the total percentage allocation in stocks is still increasing with investment horizon, although $\pi_{1}$ is not; this is illustrated in Figure 9. 


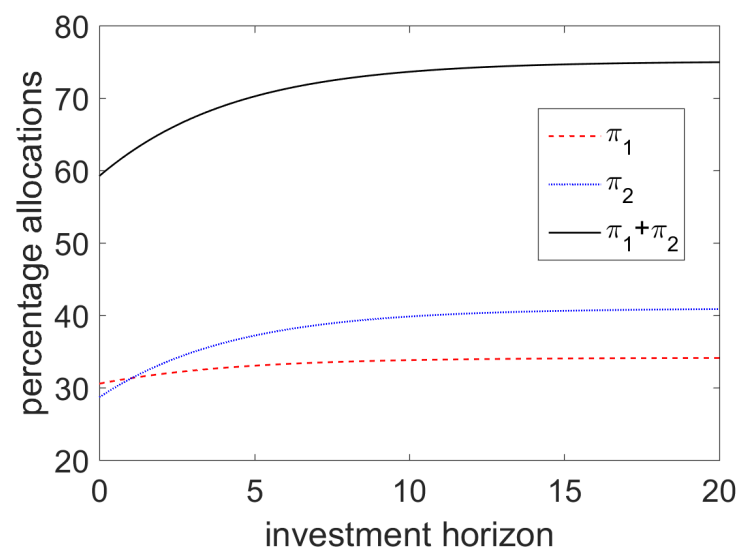

Figure 8 The optimal percentage stock allocations against investment horizon for the time-varying Gaussian mean return model with two negatively correlated stocks. Default parameter values: $\rho_{1}^{S X}=-0.15, \rho_{2}^{S X}=-0.93$, $\rho_{S}=-0.1, \sigma_{1}=0.15, \sigma_{2}=0.17, \delta_{1}=0.09, \delta_{2}=0.11$, and other parameters are the same as in Figure 4 . It can be seen that both of the percentage stock allocations are increasing with investment horizon.

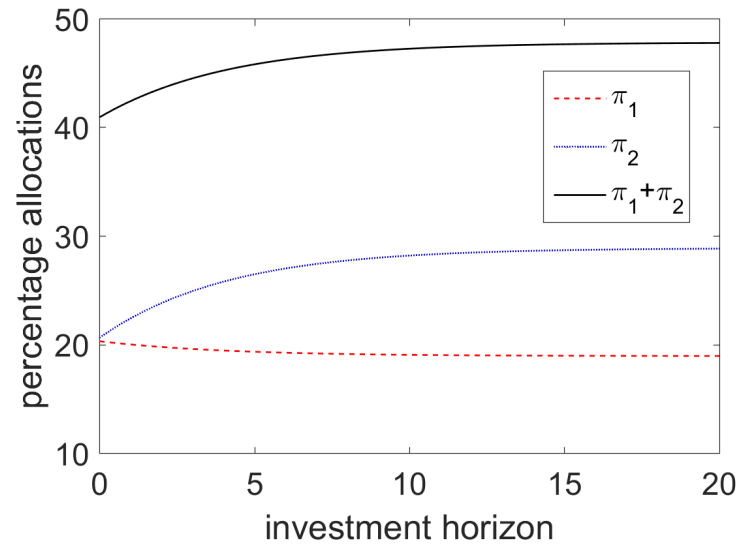

Figure 9 The optimal percentage stock allocations against investment horizon for the time-varying Gaussian mean return model with two positively correlated stocks. Default parameter values: $\rho_{S}=0.3$, and other parameters are the same as in Figure 8. It can be seen that the percentage allocation for stock $\mathbf{2}$ is increasing with investment horizon, while the percentage allocation for stock 1 is decreasing with investment horizon, but the total percentage allocation in stocks is still increasing with investment horizon.

\section{B. Monte-Carlo Simulation for Quadratic BSDEs}

In this section, we use a Monte-Carlo simulation method proposed by Chassagneux and Richou [20] to solve our quadratic BSDE (38) for the general case of multiple stocks.

The BSDE (38) is in fact the backward part of the (decoupled) forward-backward SDE, in which the forward part is the the SDE for the market state process $X$ given by (35). To simulate the BSDE (38), we have to simulate the process $X$ efficiently. To do so, we use the Markovian quantization method in Chassagneux and Richou [20], which has two components. 
The first component is to simulate the Brownian sample paths efficiently. Given a partition $\mathcal{T}=\left\{0=t_{0}<t_{1}<\cdots<t_{I}=T\right\}$ on the time interval $[0, T]$, denote $h_{i}=t_{i+1}-t_{i}$. We first simulate the $K$-dimensional Brownian increment $\Delta B_{i}^{X}$ over each subinterval $\left[t_{i}, t_{i+1}\right)$, and then use its quantization approximation $\Delta \hat{B}_{i}^{X}=\sqrt{h_{i}} G_{M}\left(\frac{\Delta B_{i}^{X}}{\sqrt{h_{i}}}\right)$ to simulate the Brownian paths. Here the function $G_{M}$ is designed to capture the standard ( $K$-dimensional) Gaussian distribution by an optimal grid with only $M$ points in $\mathbb{R}^{K}$. Pages, Pham and Printems [50] and the optimal grid data can be downloaded from the website: http://www.quantize.mathsfi.com/gaussian_database.

The second component is to project the simulated $X$ into a grid with finite points, which is constructed as follows. Let $\mathbb{Z}^{K}$ be the set of $K$-dimensional integer numbers. Given an initial value $X_{0} \in \mathbb{R}^{K}$ of the forward process $X$, we construct the following bounded grid centered at $X_{0}$ for a fixed $\hat{\delta}>0$ and a fixed positive integer $\kappa$,

$$
\left\{x \in \mathbb{R}^{K}: \frac{x-X_{0}}{\hat{\delta}} \in \mathbb{Z}^{K} \text { and } \frac{\left|x^{j}-X_{0}^{j}\right|}{\hat{\delta}} \leq \kappa, 1 \leq j \leq K\right\} .
$$

With this grid, a simulated $\mathbb{R}^{K}$-value $x=\left(x^{1}, \cdots, x^{K}\right)^{\top}$ of $X$ can be projected by an operator $\Pi(x)=\left(\Pi^{(1)}\left(x^{1}\right), \cdots, \Pi^{(K)}\left(x^{K}\right)\right)^{\top}$ with

$$
\Pi^{(j)}\left(x^{j}\right)= \begin{cases}\hat{\delta}\left\lfloor\hat{\delta}^{-1}\left(x^{j}-X_{0}^{j}\right)+\frac{1}{2}\right\rfloor+X_{0}^{j}, & \text { if }\left|x^{j}-X_{0}^{j}\right| \leq \kappa \hat{\delta} \\ \kappa \hat{\delta}+X_{0}^{j}, & \text { if } x^{j}-X_{0}^{j}>\kappa \hat{\delta} \\ -\kappa \hat{\delta}+X_{0}^{j}, & \text { if } x^{j}-X_{0}^{j}<-\kappa \hat{\delta} .\end{cases}
$$

Here, for any real number $z,\lfloor z\rfloor$ means the nearest integer less than or equal to $z$.

With these two quantization components, the Euler scheme for the forward process $X$ can be carried out by the iterations

$$
\left\{\begin{array}{l}
\hat{X}_{i+1}^{\mathcal{T}}=\Pi\left[\hat{X}_{i}^{\mathcal{T}}+h_{i} m\left(i, \hat{X}_{i}^{\mathcal{T}}\right)+\nu\left(i, \hat{X}_{i}^{\mathcal{T}}\right) \Delta \hat{B}_{i}^{X}\right], \\
\hat{X}_{0}^{\mathcal{T}}=X_{0}
\end{array}\right.
$$

Note that the truncated process $\hat{X}^{\mathcal{T}}$ is a Markovian process on $\Gamma$.

For a classical FBSDE with the Lipschitz condition, the BTZ-scheme in Bouchard and Touzi [12] and Zhang [56] uses Monte Carlo simulation and Malliavin derivative to compute a numerical solution. However the quadratic BSDE like (38) may have a quadratic growth, which violates the Lipschitz condition. Chassagneux and Richou [20] propose a truncation on 
the trouble-making component $Z$ in the generator, and modify the BTZ-scheme together with the quantization to get a numerical solution $\left(\hat{Y}^{\mathcal{T}}, \hat{Z}^{\mathcal{T}}\right)_{0 \leq i \leq I}$. Given the simulated forward process $\hat{X}_{i}^{\mathcal{T}}$, simulated Brownian increment $\Delta \hat{B}_{i}^{X}$, and a big real number $n$ for truncation, their algorithm is as follows.

(i) The terminal condition is $\left(\hat{Y}_{I}^{\mathcal{T}}, \hat{Z}_{I}^{\mathcal{T}}\right)=(0,0)$;

(ii) For $i<I$, the transition from step $i+1$ to step $i$ is given by

$$
\begin{aligned}
\hat{Y}_{i}^{\mathcal{T}} & =\mathbb{E}_{t_{i}}\left[\hat{Y}_{i+1}^{\mathcal{T}}+h_{i} g\left(t, \hat{X}_{i}^{\mathcal{T}}, \varphi_{n}\left(\hat{Z}_{i}^{\mathcal{T}}\right)\right)\right] \\
\hat{Z}_{i}^{\mathcal{T}} & =\mathbb{E}_{t_{i}}\left[\hat{Y}_{i+1}^{\mathcal{T}} \Lambda_{i}^{R}\right]
\end{aligned}
$$

where $\left(\Lambda_{i}^{R}\right)^{l}=\frac{-R}{\sqrt{h_{i}}} \vee \frac{\left(\Delta \hat{B}_{i}^{X}\right)^{l}}{\sqrt{h_{i}}} \wedge \frac{R}{\sqrt{h_{i}}}$, with $R \geq \ln (n)$, and $1 \leq l \leq K$; the truncated function $\varphi_{n}$ is defined as $\varphi_{n}(z)=\left\{\begin{array}{ll}z, & \text { if }|z| \leq n \\ z \frac{|z| \wedge n}{|z|}, & \text { if }|z|>n\end{array}\right.$.

Chassagneux and Richou [20] prove the following two propositions to ensure that the scheme works for our purpose.

Proposition 5. (Convergence of the scheme) Denote $(Y, Z)$ to be the solution to the BSDE (38). In the algorithm if we take $\delta \sim n^{-3 / 2}, \kappa \sim n^{3 / 2+\tilde{\eta}}$ and $M \sim n^{(1+\alpha) K}$ for some $\alpha \in(0,1 / 2)$ and $\tilde{\eta}>0$, then for any $\eta>0$ there exists a constant $C_{\alpha, \eta, \tilde{\eta}}$ only depending on $\alpha, \eta, \tilde{\eta}$, such that

$$
\left|Y_{0}-\hat{Y}_{0}^{\mathcal{T}}\right| \leq C_{\alpha, \eta, \tilde{\eta}} h^{1 / 2-\eta}
$$

Proposition 6. (Markovian property of the solutions ) We have $\left(\hat{Y}^{\mathcal{T}}, \hat{Z}^{\mathcal{T}}\right)$ is a Markovian process. More precisely, for all $i \in\{0, \ldots, I\}$, there exist two sequences of functions $u^{\mathcal{T}}\left(t_{i}, \cdot\right): \Gamma \mapsto \mathbb{R}$ and $v^{\mathcal{T}}\left(t_{i}, \cdot\right): \Gamma \mapsto \mathbb{R}^{1 \times K}$ such that

$$
\hat{Y}_{i}^{\mathcal{T}}=U^{\mathcal{T}}\left(t_{i}, \hat{X}_{i}^{\mathcal{T}}\right) \text { and } \hat{Z}_{i}^{\mathcal{T}}=V^{\mathcal{T}}\left(t_{i}, \hat{X}_{i}^{\mathcal{T}}\right)
$$


with terminal conditions given by $u^{\mathcal{T}}\left(t_{I}, x\right)=0$ and $v^{\mathcal{T}}\left(t_{I}, x\right)=0$. The two sequences of functions can be computed on the grids by the following backward induction: for all $i \in$ $\{0, \ldots, I\}$ and $x \in \Gamma$

$$
\left\{\begin{aligned}
V^{\mathcal{T}}\left(t_{i}, x\right)= & \mathbb{E}\left[U^{\mathcal{T}}\left(t_{i+1}, \Pi\left(x+h_{i} m\left(t_{i}, x\right)+\sqrt{h_{i}} \nu\left(t_{i}, x\right) G_{M}(\xi)\right)\right) \frac{G_{M}^{R}(\xi)}{\sqrt{h_{i}}}\right], \\
U^{\mathcal{T}}\left(t_{i}, x\right)= & \mathbb{E}\left[U^{\mathcal{T}}\left(t_{i+1}, \Pi\left(x+h_{i} m\left(t_{i}, x\right)+\sqrt{h_{i}} \nu\left(t_{i}, x\right) G_{M}(\xi)\right)\right)\right] \\
& +h_{i} g_{n}\left(t_{i}, x, V^{\mathcal{T}}\left(t_{i}, x\right)\right) \quad \text { for } i<I,
\end{aligned}\right.
$$

with Gaussian distribution $\xi \sim \mathcal{N}(0,1)$ and $\left(G_{M}^{R}(\cdot)\right)^{l}=(-R) \vee\left(G_{M}(\cdot)\right)^{l} \wedge R$, for $l \in$ $\{0, \ldots, I\}$

Note that, unlike the traditional Monte Carlo simulation which usually needs to simulate many paths to calculate an expectation, the scheme (42) only involves a weighted sum with finite number $M$, which significantly reduces the amount of computation.

\section{Numerical examples}

We assume the Gaussian mean return market setting and implement the above Monte-Carlo simulation approach with $I=50, \kappa=50, \hat{\delta}=0.025, M=100, n=9, R=6$ in the following two examples.

(1) One stock and one state process.

In this case we have an explicit optimal policy as shown before. We use the parameter values estimated by Wachter $[55]: \rho=-0.93, r=0.017, \sigma=0.15, X_{0}=\bar{X}=0.273, \lambda=0.27$, and $\nu=0.065$, and take $\gamma=3$ and $T=2$. We find that compared with the explicit optimal policy, the relative error of the Monte-Carlo simulation approach is merely $0.32 \%$.

(2) Two stocks and two state processes.

The two stocks' prices are assumed to evolve according to:

$$
\begin{aligned}
& \frac{d S_{i t}}{S_{i t}}=\left(r+\sum_{j=1}^{2} \epsilon_{i j} X_{j t}\right) d t+\sum_{j=1}^{2} \sigma_{i j} d B_{j t}, \\
& d X_{i t}=\lambda_{i}\left(\bar{X}_{i}-X_{i t}\right) d t+\sum_{j=1}^{2} \nu_{i j} d B_{j t}^{X},
\end{aligned}
$$


for $i=1,2$, where $\mathbb{E}\left[d B_{i t} d B_{j t}^{X}\right]=\rho_{i j} d t$. No explicit solutions are available in this case. Using the above Monte-Carlo simulation approach, we obtain $\left(\pi_{1}, \pi_{2}\right)$, the optimal mean percentage allocations in stock 1 and stock 2, as shown in Figure 10.

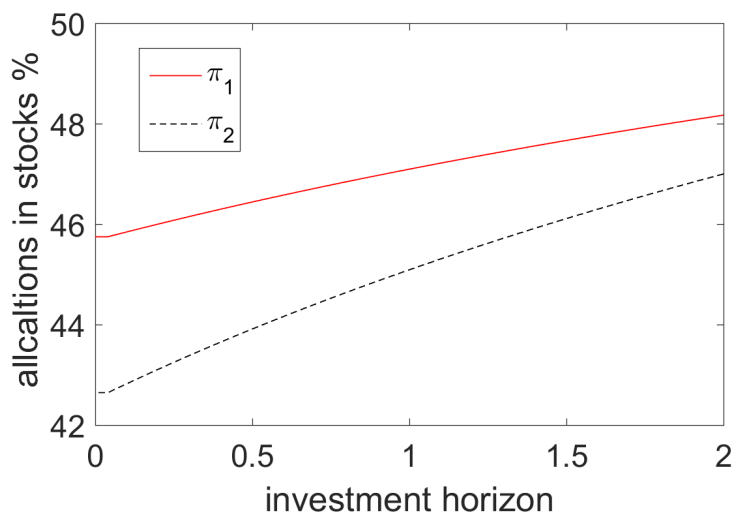

Figure 10 The optimal mean percentage allocations in stock 1 and stock 2 for our mean-variance model in the Gaussian mean return market setting (43)-(44). Here we use the Monte-Carlo simulation approach. Default parameter values: $T=2, \gamma=3, r=0.017,\left[\rho_{11}, \rho_{12} ; \rho_{21}, \rho_{22}\right]=[-0.15,-0.1 ;-0.1,-0.93]$, $\left[\sigma_{11}, \sigma_{12} ; \sigma_{21}, \sigma_{22}\right]=[0.15,0 ;-0.05,0.15],\left[X_{0,1}, X_{0,2}\right]=\left[\bar{X}_{1}, \bar{X}_{2}\right]=[0.273,0.3],\left[\lambda_{1}, \lambda_{2}\right]=[0.27,0.28]$, $\left[\nu_{11}, \nu_{12} ; \nu_{21}, \nu_{22}\right]=[0.065,0 ;-0.01,0.08],\left[\epsilon_{11}, \epsilon_{12} ; \epsilon_{21}, \epsilon_{22}\right]=[0.06,0.04 ; 0.04,0.06]$.

\section{Proofs of Theorems, Propositions and Some Assertions}

\section{C.1. Proof of Theorem 1}

If there exists a perturbation $v$ at time $t$, such that $\liminf _{h \rightarrow 0^{+}}-\frac{\operatorname{Var}_{t}\left(R_{T}^{\hat{\pi}}\right)-\operatorname{Var}_{t}\left(R_{T}^{\pi^{h, v}}\right)}{h}<0$, then

$$
\begin{aligned}
& \liminf _{h \rightarrow 0^{+}} \frac{J(t, y, x ; \hat{\pi})-J\left(t, y, x ; \pi^{h, v}\right)}{h} \\
= & \liminf _{h \rightarrow 0^{+}} \frac{\left(\mathbb{E}_{t}\left[R_{T}^{\hat{\hat{\pi}}}\right]-\mathbb{E}_{t}\left[R_{T}^{\pi^{h, v}}\right]\right)-\frac{\gamma}{2}\left(\operatorname{Var}_{t}\left(R_{T}^{\hat{\hat{\pi}}}\right)-\operatorname{Var}_{t}\left(R_{T}^{\pi^{h, v}}\right)\right)}{h} \\
\leq & \liminf _{h \rightarrow 0^{+}}-\frac{\gamma}{2} \frac{\operatorname{Var}_{t}\left(R_{T}^{\hat{\pi}}\right)-\operatorname{Var}_{t}\left(R_{T}^{\pi^{h, v}}\right)}{h}<0,
\end{aligned}
$$

which contradicts the fact that $\hat{\pi}$ is an equilibrium strategy.

\section{C.2. Proof of Theorem 2}

For any deterministic trading policy $\pi$., we can get $\mathbb{E}_{t}\left[R_{T}\right]=R_{t}+$ $\int_{t}^{T}\left(r+\pi_{s}(\mu-r)-\frac{1}{2} \pi_{s}^{2} \sigma^{2}\right) d s$ and $\operatorname{Var}_{t}\left(R_{T}\right)=\int_{t}^{T} \pi_{s}^{2} \sigma^{2} d s$. Since $\hat{\pi}$ is deterministic,

$$
J\left(t, R_{t}, X_{t} ; \hat{\pi}\right)=R_{t}+\int_{t}^{T}\left(r+\hat{\pi}_{s}(\mu-r)-\frac{1+\gamma}{2}\left(\hat{\pi}_{s}\right)^{2} \sigma^{2}\right) d s .
$$


Similarly, for any perturbation $\pi_{s}^{h, v}:=v \mathbf{1}_{s \in[t, t+h)}+\hat{\pi}_{s} \mathbf{1}_{s \in[t+h, T]}$, which is also deterministic, we also have

$$
\begin{aligned}
& J\left(t, R_{t}, X_{t} ; \pi^{h, v}\right)=R_{t}+\int_{t}^{T}\left(r+\pi_{s}^{h, v}(\mu-r)-\frac{1+\gamma}{2}\left(\pi_{s}^{h, v}\right)^{2} \sigma^{2}\right) d s \\
= & J\left(t, R_{t}, X_{t} ; \hat{\pi}\right)+\int_{t}^{t+h}\left(\left[v(\mu-r)-\frac{1+\gamma}{2} v^{2} \sigma^{2}\right]-\left[\hat{\pi}(\mu-r)-\frac{1+\gamma}{2}\left(\hat{\pi}_{s}\right)^{2} \sigma^{2}\right]\right) d s .
\end{aligned}
$$

Since $\hat{\pi}_{s}=\operatorname{argmax}_{v \in \mathbb{R}}\left\{v(\mu-r)-\frac{1+\gamma}{2} v^{2} \sigma^{2}\right\}$, we know $J\left(t, R_{t}, X_{t} ; \pi^{h, v}\right) \leq J\left(, R_{t}, X_{t} ; \hat{\pi}\right)$. Hence $\hat{\pi}$ is an equilibrium solution. Part (ii) then follows by a direct calculation.

\section{C.3. Proofs of Theorem 3 , and Propositions 3 and 4}

Let us first consider the general multi-asset market described in Online Supplement A. Furthermore, we impose a constraint on the proportional portfolio: $\pi_{t} \in \Pi_{t}$ for some $\mathcal{F}_{t}^{B^{X}}$ adapted closed convex subset $\Pi_{t} \subseteq \mathbb{R}^{n}$ with $0 \in \Pi_{t}$, where $r_{t}=r\left(t, X_{t}\right), \mu_{t}=\mu\left(t, X_{t}\right)$, $\sigma_{t}=\sigma\left(t, X_{t}\right), m_{t}=m\left(t, X_{t}\right), a n d \nu_{t}=\nu\left(t, X_{t}\right)$. Recall $\theta_{t}:=\theta\left(t, X_{t}\right)=\sigma_{t}^{-1}\left(\mu_{t}-r_{t}\right)$. Denote $a(t, \pi)=r_{t}+\frac{\left|\theta_{t}\right|^{2}}{2}-\frac{1}{2}\left|\sigma_{t}^{\top} \pi-\theta_{t}\right|^{2}$. For later use, we define for any $z \in \mathbb{R}^{K}, \hat{v}(t, z)=$ $\operatorname{argmin}_{\pi \in \Pi_{t}}\left|\sigma_{t}^{\top} \pi-\theta_{t}\right|^{2}+\gamma\left|\sigma_{t}^{\top} \pi+\rho z\right|^{2}$ and $g(t, z):=a(t, \hat{v}(t, z))=r_{t}+\frac{1}{2}\left|\theta_{t}\right|^{2}-\frac{1}{2} \mid \sigma_{t}^{\top} \hat{v}(t, z)-$ $\left.\theta_{t}\right|^{2}$. It is easy to see

$$
\begin{aligned}
|g(t, z)| & \leq\left|r_{t}\right|+\frac{1}{2}\left|\theta_{t}\right|^{2}+\frac{1}{2}\left(\left|\sigma_{t}^{\top} \hat{v}(t, z)-\theta_{t}\right|^{2}+\gamma\left|\sigma_{t}^{\top} v+\rho z\right|^{2}\right) \\
& \leq\left|r_{t}\right|+\frac{1}{2}\left|\theta_{t}\right|^{2}+\frac{1}{2}\left(\left|\theta_{t}\right|^{2}+\gamma|\rho z|^{2}\right),
\end{aligned}
$$

by which we say $g$ is quadratic dominated.

We now give a general result.

Theorem 4. Suppose $\mathbb{E}\left[\int_{0}^{T}\left|\theta_{t}\right|^{2} d t\right]<+\infty$. Consider the BSDE (38) with $g(\cdot, \cdot)$ being as given in (45).

(i) If the BSDE admits a solution $(Y, Z)$ in the classical sense. Then $\pi_{t}=\hat{v}\left(t, Z_{t}\right)$ is an equilibrium policy with the expected annual return rate $\frac{1}{T-t} \mathbb{E}_{t}\left[\hat{R}_{T}-\hat{R}_{t}\right]=\frac{Y_{t}}{T-t}$. 
(ii) If $\Pi_{t}=\Pi\left(t, X_{t}\right)$ for some deterministic set-value function $\Pi$, and the solution $(Y, Z)$ to (38) is unique, then there exists a deterministic function $f(\cdot, \cdot)$ such that $Y_{t}=f\left(t, X_{t}\right)$. Further, if $f$ is $C^{1,2}$, then $f$ satisfies the PDE

$$
0=\frac{\partial f}{\partial t}+\left(\frac{\partial f}{\partial x}\right)^{\top} m(t, x)+\frac{1}{2} \operatorname{Tr}\left(\nu(t, x)^{\top} \frac{\partial^{2} f}{\partial x^{2}} \nu(t, x)\right)+g\left(t, Z_{t}\right)
$$

with the terminal condition $f(T, x, s)=0, Z_{t}=\nu(t, x)^{\top} \frac{\partial f}{\partial x}(t, x, s)$, and the equilibrium policy is given by $\hat{\pi}\left(t, R_{t}, X_{t}\right)=\hat{\pi}_{t}:=\hat{v}\left(t, Z_{t}\right)$.

\section{Proof}

(i) Given $\mathbb{E}\left[\int_{0}^{T}\left|\theta_{t}\right|^{2} d t\right]<+\infty$, we can easily see that

$$
\begin{aligned}
\mathbb{E}\left[\int_{0}^{T}\left|\sigma_{t}^{\top} \hat{\pi}_{t}\right|^{2} d t\right] & \leq \mathbb{E}\left[\int_{0}^{T}\left(\left|\sigma_{t}^{\top} \hat{\pi}_{t}-\theta_{t}\right|^{2}+\gamma\left|\sigma_{t}^{\top} \hat{\pi}+\rho Z\right|^{2}+\left|\theta_{t}\right|^{2}\right) d t\right] \\
& \leq \mathbb{E}\left[\int_{0}^{T}\left(\left|\theta_{t}\right|^{2}+\gamma|\rho Z|^{2}+\left|\theta_{t}\right|^{2}\right) d t\right]<+\infty .
\end{aligned}
$$

Hence $\hat{\pi}\left(t, R_{t}, X_{t}\right) \equiv \hat{\pi}_{t}$ is admissible. Denote by $\hat{W}$. its wealth process and $\hat{R}_{t}=\ln \hat{W}_{t}$. We can assume that $Z_{t}$ is right continuous, or simply take its right continuous version. By the definition of the solution to the BSDE and the fact that $g$ is quadratic dominiated, we know $\mathbb{E}\left[\int_{0}^{T} g\left(t, Z_{t}\right)^{2} d s\right]<+\infty$. By the BSDE (38), we know $M_{t}:=Y_{t}+\int_{0}^{t} g\left(s, Z_{s}\right) d s$ is a martingale with $M_{T}=\int_{0}^{T} g\left(s, Z_{s}\right) d s$, so for any $t \in[0, T]$, we have

$$
\left.Y_{t}+\int_{0}^{t} g\left(s, Z_{s}\right) d s=M_{t}=\mathbb{E}_{t}\left[\int_{0}^{T} g\left(s, Z_{s}\right) d s\right]=\int_{0}^{t} g\left(s, Z_{s}\right) d s\right]+\mathbb{E}_{t}\left[\int_{t}^{T} g\left(s, Z_{s}\right) d s\right],
$$

which gives $Y_{t}=\mathbb{E}_{t}\left[\int_{t}^{T} g\left(s, Z_{s}\right) d s\right]=\mathbb{E}_{t}\left[\hat{R}_{T}-\hat{R}_{t}\right]$. For any local perturbation $\pi_{s}^{h, v}=$ $\hat{\pi}_{s} \mathbf{1}_{s \notin[t, t+h)}+v \mathbf{1}_{s \in[t, t+h)}$, denote its log return process as $R_{\text {. }}^{h, v}$, then $R_{T}^{h, v}-R_{t+h}^{h, v}=\hat{R}_{T}-\hat{R}_{t+h}$, which implies $\mathbb{E}_{t+h}\left[R_{T}^{h, v}\right]=R_{t+h}^{h, v}+Y_{t+h}$. It follows

$$
\begin{aligned}
& J\left(t, R_{t}, X_{t}, S_{t} ; \pi^{h, v}\right)=\mathbb{E}_{t}\left[R_{T}^{h, v}\right]-\frac{\lambda}{2} \operatorname{Var}_{t}\left(R_{T}^{h, v}\right) \\
= & \mathbb{E}_{t}\left[\mathbb{E}_{t+h}\left[R_{T}^{h, v}\right]-\frac{\lambda}{2} \operatorname{Var}_{t+h}\left(R_{T}^{h, v}\right)\right]-\frac{\lambda}{2} \operatorname{Var}_{t}\left(\mathbb{E}_{t+h}\left[R_{T}^{h, v}\right]\right) \\
= & \mathbb{E}_{t}\left[R_{t+h}^{h, v}+Y_{t+h}-\frac{\lambda}{2} \operatorname{Var}_{t+h}\left(R_{T}^{h, v}-R_{t+h}^{h, v}\right)\right]-\frac{\lambda}{2} \operatorname{Var}_{t}\left(R_{t+h}^{h, v}+Y_{t+h}\right) \\
= & \mathbb{E}_{t}\left[\hat{R}_{t+h}+Y_{t+h}-\frac{\lambda}{2} \operatorname{Var}_{t+h}\left(\hat{R}_{T}-\hat{R}_{t+h}\right)\right]+\mathbb{E}_{t}\left[R_{t+h}^{h, v}-\hat{R}_{t+h}\right]-\frac{\lambda}{2} \operatorname{Var}_{t}\left(R_{t+h}^{h, v}+Y_{t+h}\right) \\
= & J\left(t, R_{t}, X_{t}, S_{t} ; \hat{\pi}\right)+\mathbb{E}_{t}\left[R_{t+h}^{h, v}-\hat{R}_{t+h}\right]-\frac{\lambda}{2} \operatorname{Var}_{t}\left(R_{t+h}^{h, v}+Y_{t+h}\right) .
\end{aligned}
$$


On one hand, $\mathbb{E}_{t}\left[R_{t+h}^{h, v}-\hat{R}_{t+h}\right]=\int_{t}^{t+h} \mathbb{E}_{t}\left[a(s, v)-a\left(s, \hat{\pi}_{s}\right)\right] d s$. On the other hand, noting that $R_{t+h}^{h, v}-R_{t}=\int_{t}^{t+h} a(s, v) d s+\int_{t}^{t+h} v^{\top} \sigma_{s} d B_{s}$ and $Y_{t+h}=Y_{t}-\int_{t}^{t+h} a\left(s, \hat{\pi}_{s}\right) d s+\int_{t}^{t+h} Z_{s}^{\top} d B_{s}^{X}$,

we get

$$
R_{t+h}^{h, v}+Y_{t+h}=R_{t}+Y_{t}+\int_{t}^{t+h}\left[a(s, v)-a\left(s, \hat{\pi}_{s}\right)\right] d s+\int_{t}^{t+h}\left(\sigma_{t}^{\top} v\right)^{\top} d B_{s}+\int_{t}^{t+h} Z_{s}^{\top} d B_{s}^{X} .
$$

It is not hard to see that $\mathbb{E}_{t}\left[\int_{t}^{T}\left|a(s, v)-a\left(s, \hat{\pi}_{s}\right)\right| d s\right]<+\infty$, hence

$$
\operatorname{Var}_{t}\left(R_{t+h}^{h, v}+Y_{t+h}\right)=\mathbb{E}_{t}\left[\int_{t}^{t+h} \phi\left(s, \sigma_{s} v\right) d s\right]+o(h)
$$

where $\phi(s, x):=|x|^{2}+\left|Z_{s}\right|^{2}+2 x^{\top} \rho Z_{s}$. Then

$$
\begin{aligned}
& \lim _{h \downarrow 0} \frac{J\left(t, R_{t}, X_{t}, S_{t} ; \pi^{h, v}\right)-J\left(t, R_{t}, X_{t}, S_{t} ; \hat{\pi}\right)}{h} \\
= & \lim _{h \downarrow 0} \frac{\mathbb{E}_{t}\left[\int_{t}^{t+h} a(s, v)-a\left(s, \hat{\pi}_{s}\right)-\frac{\gamma}{2} \phi\left(s, \sigma_{s}^{\top} v\right)+\frac{\gamma}{2} \phi\left(s, \sigma_{s}^{\top} \hat{\pi}_{s}\right) d s\right]}{h} \\
= & a(t, v)-\frac{\gamma}{2} \phi\left(t, \sigma_{t}^{\top} v\right)-a\left(t, \hat{\pi}_{t}\right)+\frac{\gamma}{2} \phi\left(t, \sigma_{t}^{\top} \hat{\pi}_{t}\right) .
\end{aligned}
$$

Note that $a(t, v)-\frac{\gamma}{2} \phi\left(t, \sigma_{t}^{\top} v\right)=r_{t}+\frac{1}{2}\left|\theta_{t}\right|^{2}-\frac{1}{2}\left|\sigma_{t}^{\top} v-\theta_{t}\right|^{2}-\frac{\gamma}{2}\left(\left|\sigma_{t}^{\top} v\right|^{2}+\left|Z_{s}\right|^{2}+2 v^{\top} \sigma_{t} \rho Z_{t}\right)$, whose maximal value for $v \in \Pi$ is achieved at $\hat{\pi}_{t}$. This implies that $\hat{\pi}$ is an equilibrium policy.

(ii) In this case the BSDE (38) is a Markovian system, hence we can write $Y_{t}=f\left(t, X_{t}\right)$. If $f$ is $C^{1,2}$, then we can apply Itô's formula to get the PDE (46) and $Z$ as presented in the theorem. The desired result then follows.

From Theorem 4 we immediately have the following corollary.

Corollary 2. Assume the same condition in Proposition 4 .

(i) If $\Pi_{t} \equiv \mathbb{R}^{n}$, then

$$
\hat{v}(t, z)=\frac{1}{1+\gamma}\left(\sigma_{t}^{\top}\right)^{-1}[\theta-\gamma \rho z], \quad g(t, z)=r_{t}+\frac{1}{2}\left|\theta_{t}\right|^{2}-\frac{1}{2} \frac{\gamma^{2}}{(1+\gamma)^{2}}\left|\theta_{t}+\rho z\right|^{2}
$$

and the BSDE (38) for $Y$ admits a unique solution.

(ii) If $N=K=1$ and $\Pi_{t}=\left[\underline{\pi}_{t}, \bar{\pi}_{t}\right]$ for some $\mathcal{F}_{t}^{B^{X}}$-adapted processes $\underline{\pi}_{t}$ and $\bar{\pi}_{t}$, then

$$
\hat{v}(t, z)=\underline{\pi}_{t} \vee\left(\frac{1}{1+\gamma} \sigma_{t}^{-1}[\theta-\gamma \rho z]\right) \wedge \bar{\pi}_{t}
$$




$$
g(t, z)=r_{t}+\frac{1}{2} \theta_{t}^{2}-\frac{1}{2}\left(\left(\theta_{t}-\sigma_{t} \underline{\pi}_{t}\right) \wedge \frac{\gamma\left(\theta_{t}+\rho z\right)}{1+\gamma} \vee\left(\theta_{t}-\sigma_{t} \bar{\pi}_{t}\right)\right)^{2} .
$$

It is easy to see that Proposition 3 and Proposition 4 are exactly part (ii) and part (i) of Corollary 2, respectively, and the existence and uniqueness of the solution to the BSDE (38) can be deduced from Briand and $\mathrm{Hu}[15]$. Finally, Theorem 3 is a special case of Proposition 4 (other than part (ii), whose proof is given after this corollary), where the regularity condition for $\theta$ is weakened in terms of the explicit solution to the BSDE as given in the theorem.

It remains to prove part (ii) in Theorem 3. We rewrite the function $g$ as

$$
\begin{aligned}
g(t, z) & =r_{t}+\frac{1-k^{2}}{2}\left|\theta_{t}\right|^{2}-\frac{k^{2}}{2}\left(2 z \theta_{t}^{\top} \rho+z^{2}|\rho|^{2}\right) \\
& =\alpha\left(t, X_{t}\right)-\frac{|k \rho|^{2}}{2}\left(z+\beta\left(t, X_{t}\right)\right)^{2} .
\end{aligned}
$$

Define $\hat{Z}_{t}=Z_{t}+\beta\left(t, X_{t}\right)$, and $M_{t}=Y_{t}+\int_{0}^{t} \alpha\left(s, X_{s}\right) d s+\int_{0}^{t} \beta\left(s, X_{s}\right) d B_{s}^{X}$. Then

$$
d M_{t}=\frac{|k \rho|^{2}}{2} \hat{Z}_{t}^{2} d t+\hat{Z}_{t} d B_{t}^{X}, \quad M_{T}=\int_{0}^{T} \alpha\left(t, X_{t}\right) d t+\int_{0}^{T} \beta\left(t, X_{t}\right) d B_{t}^{X} .
$$

It is easy to check that $M_{t}=\frac{-1}{|k \rho|^{2}} \ln \mathbb{E}_{t}\left[e^{-|k \rho|^{2}\left(\int_{0}^{T} \alpha\left(s, X_{s}\right) d s+\int_{0}^{T} \beta\left(s, X_{s}\right) d B_{s}^{X}\right)}\right]$ with $e^{-|k \rho|^{2} M_{t}}$ being a martingale. So

$$
Y_{t}=\frac{-1}{|k \rho|^{2}} \ln \mathbb{E}_{t}\left[e^{-|k \rho|^{2}\left(\int_{t}^{T} \alpha\left(s, X_{s}\right) d s+\int_{t}^{T} \beta\left(s, X_{s}\right) d B_{s}^{X}\right)}\right]
$$

\section{C.4. Proof of Proposition 1}

Using a transformation, $f(t, x)=-\frac{(1+\gamma)^{2}}{\gamma^{2} \rho^{2}} \ln u(t, x)+r(T-t)$, PDE (18) becomes

$$
\frac{\partial u}{\partial t}+\left(m(x)-\frac{\gamma^{2} \rho \nu(x)}{(1+\gamma)^{2}} \theta(t, x)\right) \frac{\partial u}{\partial x}+\frac{1}{2} \nu(x)^{2} \frac{\partial^{2} u}{\partial x^{2}}-\frac{(2 \gamma+1) \gamma^{2} \rho^{2}}{2(\gamma+1)^{4}} \theta(t, x)^{2} u=0
$$

with terminal condition $u(T, x)=1$.

For the stochastic volatility model, PDE (48) reduces to

$$
\frac{\partial u}{\partial t}+(\lambda \bar{X}-\bar{b} x) \frac{\partial u}{\partial x}+\frac{1}{2} \bar{\nu}^{2} x \frac{\partial^{2} u}{\partial x^{2}}-\bar{c} x u=0
$$

where $\bar{c}=\frac{(2 \gamma+1) \gamma^{2} \rho^{2} \delta^{2}}{2(\gamma+1)^{4}} \geq 0$, and $\bar{b}$ is as given in (23). We assert $u(t, x)=\exp \{g(t) x+h(t)\}$. Indeed, using (49), we deduce the following ordinary differential equation (ODE) system:

$$
\begin{cases}g_{t}-\bar{b} g+\frac{1}{2} \bar{\nu}^{2} g^{2}-\bar{c}=0, & g(T)=0 \\ h_{t}+\lambda \bar{X} g=0, & h(T)=0\end{cases}
$$


Let $\bar{q}$ be as given in (23). Owing to $\bar{q} \geq|\bar{b}|$, it is easy to verify

$$
g(t)=\frac{-2 \bar{c}\left(e^{\bar{q}(T-t)}-1\right)}{(\bar{b}+\bar{q})\left(e^{\bar{q}(T-t)}-1\right)+2 \bar{q}} \leq 0 .
$$

Note that $f(t, x)=-\frac{(1+\gamma)^{2}}{\gamma^{2} \rho^{2}}[g(t) x+h(t)]$. Hence

$$
\frac{\partial f}{\partial x}=-\frac{(1+\gamma)^{2}}{\gamma^{2} \rho^{2}} g(t)=\frac{(2 \gamma+1) \delta^{2}}{(\gamma+1)^{2}} \frac{e^{\bar{q}(T-t)}-1}{(\bar{b}+\bar{q})\left(e^{\bar{q}(T-t)}-1\right)+2 \bar{q}} \geq 0 .
$$

Plugging this into (14) yields (22).

Note that $\hat{\pi}\left(t, X_{t}\right)=\frac{\delta}{(1+\gamma)} X_{t}^{\frac{\alpha-1}{2 \alpha}} I$, where

$$
I=1-\frac{(2 \gamma+1) \gamma \rho \bar{\nu} \delta}{(1+\gamma)^{2}} \frac{e^{\bar{q}(T-t)}-1}{(\bar{b}+\bar{q})\left(e^{\bar{q}(T-t)}-1\right)+2 \bar{q}} .
$$

If $\rho \delta \leq 0$, then obviously $I \geq 0$. For $\rho \delta>0$,

$$
\begin{aligned}
I & \geq \frac{e^{\bar{q}(T-t)}\left[(1+\gamma)^{2}(\bar{b}+\bar{q})-(2 \gamma+1) \gamma \rho \bar{\nu} \delta\right]}{(1+\gamma)^{2}\left[(\bar{b}+\bar{q})\left(e^{\bar{q}(T-t)}-1\right)+2 \bar{q}\right]} \geq \frac{(1+\gamma)^{2} \bar{q}-(\gamma+1) \gamma \rho \bar{\nu} \delta}{(1+\gamma)^{2}\left[(\bar{b}+\bar{q})\left(e^{\bar{q}(T-t)}-1\right)+2 \bar{q}\right]} \\
& \geq \frac{(\gamma+1) \gamma(\lambda+\rho \bar{\nu} \delta)-(\gamma+1) \gamma \rho \bar{\nu} \delta}{(1+\gamma)^{2}\left[(\bar{b}+\bar{q})\left(e^{\bar{q}(T-t)}-1\right)+2 \bar{q}\right]} \geq 0 .
\end{aligned}
$$

Hence, part (i) holds. Part (ii) follows by part (i) and the property of function $X_{t}^{\frac{\alpha-1}{2 \alpha}}$. Part (iii) is obvious.

It remains to prove part (iv). Note that

$$
\frac{e^{\bar{q}(T-t)}-1}{(\bar{b}+\bar{q})\left(e^{\bar{q}(T-t)}-1\right)+2 \bar{q}}=\frac{1}{\bar{b}+\bar{q}}\left[1-\frac{2 \bar{q}}{(\bar{b}+\bar{q})\left(e^{\bar{q}(T-t)}-1\right)+2 \bar{q}}\right]
$$

is increasing in $T-t$, which yields the desired result.

\section{C.5. Proof of Proposition 2}

For this model, PDE (48) reduces to

$$
\frac{\partial u}{\partial t}+(\lambda \bar{X}-b x) \frac{\partial u}{\partial x}+\frac{1}{2} \nu^{2} \frac{\partial^{2} u}{\partial x^{2}}-c x^{2} u=0
$$

with $u(T, x)=1$, where $c=\frac{(2 \gamma+1) \gamma^{2} \rho^{2}}{2(\gamma+1)^{4}}$ and $b$ is as given in (29). We assert that $V(t, x)=$ $\exp \left\{\Theta(t) x^{2}+\Psi(t) x+\Phi(t)\right\}$. Indeed, substituting this form of $V$ into the PDE (50) gives

$$
\left\{\begin{array}{l}
\Theta_{t}-2 b \Theta+2 \nu^{2} \Theta^{2}-c=0, \quad \Theta(T)=0 \\
\Psi_{t}+\left(2 \nu^{2} \Theta-b\right) \Psi+2 \lambda \bar{X} \Theta=0, \Psi(T)=0 \\
\Phi_{t}+\lambda \bar{X} \Psi+\frac{1}{2} \nu^{2}\left(\Psi^{2}+2 \Theta\right)=0, \Phi(T)=0 .
\end{array}\right.
$$


From the first equation of $(51)$, we get

$$
\Theta(t)=\frac{-c\left(e^{2 q(T-t)}-1\right)}{(b+q)\left(e^{2 q(T-t)}-1\right)+2 q},
$$

where $q$ is as given in (29). Owing to $q \geq|b|$, we have $\Theta \leq 0$. Solving the second equation of (51) gives

$$
\Psi(t)=\frac{-2 \lambda \bar{X} c\left(e^{q(T-t)}-1\right)^{2}}{q\left[(b+q)\left(e^{2 q(T-t)}-1\right)+2 q\right]} .
$$

Plugging $\frac{\partial f}{\partial x}=-\frac{(1+\gamma)^{2}}{\gamma^{2} \rho^{2}}[\Psi(t)+2 \Theta(t) x]$ into (14) leads to the desired result.

(i) Note that $\hat{\pi}\left(t, X_{t}\right)=X_{t} \cdot I+\frac{\nu(\gamma+1)}{\rho \sigma \gamma} \Psi$, where

$$
I=\frac{1}{\sigma(1+\gamma)}+\frac{2 \nu(\gamma+1)}{\rho \sigma \gamma} \Theta
$$

Hence, $\hat{\pi}$ is increasing in $X_{t}$ if $I \geq 0$. Obviously, $I \geq 0$ when $\rho<0$. For $\rho>0$, we denote

$$
D=(b+q)\left(e^{2 q(T-t)}-1\right)+2 q
$$

By the expressions of $b, c, q$ and $\Theta$, we have

$$
\begin{aligned}
I & =\frac{1}{\sigma(1+\gamma)^{3} D}\left[e^{2 q(T-t)}\left((1+\gamma)^{2}(q+\lambda)-(\gamma+1) \gamma \rho \nu\right)+(1+\gamma)^{2}(q-b)+(2 \gamma+1) \gamma \rho \nu\right] \\
& \geq \frac{1}{\sigma(1+\gamma)^{3} D} e^{2 q(T-t)}\left((1+\gamma) \sqrt{\lambda^{2}(2 \gamma+1)+\gamma^{2}(\lambda+\rho \nu)^{2}}-(\gamma+1) \gamma \rho \nu\right) \\
& \geq \frac{1}{\sigma(1+\gamma)^{2} D} \gamma(|\lambda+\rho \nu|-\rho \nu) \geq 0 .
\end{aligned}
$$

This is desired.

(ii) From (28), obviously $\mathbb{E} \hat{\pi}>0$ when $\rho<0$. It remains to show the case $\rho>0$. Note that $(1+\gamma)^{2} q^{2} \geq \gamma^{2} \rho \nu \lambda$. Then

$$
\begin{aligned}
\mathbb{E} \hat{\pi} & =\frac{\bar{X}}{\sigma(1+\gamma)^{3} q D}\left\{2 \lambda(2 \gamma+1) \gamma \rho \nu e^{q(T-t)}+(1+\gamma)^{2} q(q-b)+(2 \gamma+1) \gamma \rho \nu(q-\lambda)+e^{2 q(T-t)} \hat{I}\right\} \\
& \geq \frac{\bar{X}}{\sigma(1+\gamma)^{3} q D} e^{2 q(T-t)} \hat{I} \geq 0
\end{aligned}
$$

where

$$
\begin{aligned}
& \hat{I}=(1+\gamma)^{2} q(b+q)-(2 \gamma+1) \gamma \rho \nu(\lambda+q) \geq(1+\gamma)^{2} q\left(\frac{\gamma^{2} \rho \nu}{(1+\gamma)^{2}}+q\right)-\gamma^{2} \rho \nu(\lambda+q) \\
& =(1+\gamma)^{2} q^{2}-\gamma^{2} \rho \nu \lambda \geq 0,
\end{aligned}
$$


which is desired.

(iii) It follows by $\Psi<0, \Theta<0$, and $\mathbb{E}\left[\hat{H}\left(t, X_{t}\right)\right]=\frac{\nu(\gamma+1)}{\rho \sigma \gamma}(\Psi+2 \Theta \bar{X})$.

(iv) Note that $\left|\mathbb{E}\left[\hat{H}\left(t, X_{t}\right)\right]\right|=\frac{(2 \gamma+1) \gamma|\rho \nu \bar{X}|}{(\gamma+1)^{3} \sigma} \tilde{I}$, where

$$
\tilde{I}=\frac{1}{q D}\left[q\left(e^{2 q(T-t)}-1\right)+\lambda\left(e^{q(T-t)}-1\right)^{2}\right]=\frac{1}{q}\left\{\frac{\lambda+q}{b+q}-h(z)\right\},
$$

with $z=e^{q(T-t)}, C_{1}=\frac{(q+\lambda)(q-b)}{q+b}+(q-\lambda), C_{2}=\frac{q-b}{q+b}$, and $h(z)=\frac{2 \lambda z+C_{1}}{z^{2}+C_{2}}$. Note that $q \geq b$, $q \geq \lambda$, and $h^{\prime}(z)=\frac{-2 \lambda z^{2}-2 C_{1} z+2 \lambda C_{2}}{\left(z+C_{2}\right)^{2}}$. Denote $\phi(z)=-2 \lambda z^{2}-2 C_{1} z+2 \lambda C_{2}$. Notice $\phi(1)=$ $-\frac{4 q^{2}}{q+b}<0$ and $\frac{-C_{1}}{2 \lambda}<0$. Hence $h^{\prime}(z)<0$ for $z \geq 1$. It follows that $-h\left(e^{q(T-t)}\right)$ and $\tilde{I}$ are increasing in $T-t$. This yields the desired result.

\section{C.6. Proof of Equivalence between Dynamic Mean-Variance and CRRA Preferences}

Denote $v_{t}=-\rho \gamma \nu\left(t, X_{t}\right) \frac{\partial f\left(t, X_{t}\right)}{\partial X}$, then $\sigma\left(t, X_{t}\right) \hat{\pi}\left(t, X_{t}\right)=\frac{1}{1+\gamma}\left(\theta\left(t, X_{t}\right)+v_{t}\right)$. We decompose $B_{t}$ into $B_{t}=\rho B_{t}^{X}+\sqrt{1-\rho^{2}} B_{t}^{\perp}$ with $B_{t}^{\perp}$ being a standard BM independent with $B$.

We use the notation $a(t, \pi), \hat{R}$. and $Y_{t}$ as in Theorem 4. Denote $a_{t}=a\left(t, \hat{\pi}_{t}\right)$. Since $Y_{t}=$ $f\left(t, X_{t}\right)$ and $Y_{t}+\int_{0}^{t} a_{s} d s$ (as in Theorem 4 ) is a martingale, hence $d Y_{t}+a_{t} d t=\frac{\partial f}{\partial x} \nu\left(t, X_{t}\right) d B^{X}$. This gives

$$
\int_{0}^{T} a_{s} d s=Y_{0}+\int_{0}^{T} \frac{\partial f\left(s, X_{s}\right)}{\partial X} \nu\left(s, X_{s}\right) d B_{s}^{X} .
$$

Define $\mathbb{Q}^{v}$ by $\frac{d \mathbb{Q}^{v}}{d \mathbb{P}}=e^{-\int_{0}^{T}\left(\frac{v_{t}}{\rho}\right)^{2} / 2 d t+\int_{0}^{T} \frac{v_{t}}{\rho} d B_{t}^{X}}$, then $\left(B_{t}^{\perp}, B^{X}-\int_{0}^{t} \frac{v_{s}}{\rho} d s\right)$ is a standard $\mathbb{Q}^{v}-\mathrm{BM}$, and hence $B_{t}^{v}:=B_{t}^{X}-\int_{0}^{t} \frac{v_{s}}{\rho} d s$ is a $\mathbb{Q}^{v}-\mathrm{BM}$.

Note that

$$
\frac{d \mathbb{Q}^{v}}{d \mathbb{P}}=e^{-\int_{0}^{T}\left(\gamma \nu\left(t, X_{t}\right) \frac{\partial f\left(t, X_{t}\right)}{\partial X}\right)^{2} / 2 d t-\int_{0}^{T}\left(\gamma \nu\left(t, X_{t}\right) \frac{\partial f\left(t, X_{t}\right)}{\partial X}\right) d B_{t}^{X}}=e^{-\int_{0}^{T}\left(\gamma \nu\left(t, X_{t}\right) \frac{\partial f\left(t, X_{t}\right)}{\partial X}\right)^{2} / 2 d t-\gamma \int_{0}^{T} a_{t} d t+\gamma Y_{0}} .
$$

Define $A_{T}:=e^{\gamma \int_{0}^{T}\left[\hat{\pi}_{t} \sigma_{t}\left(\theta_{t}+v_{t}\right)-(1+\gamma)\left|\sigma_{t} \hat{\pi}_{t}\right|^{2} / 2\right] d t}$. Recall the $\varepsilon=e^{-\frac{\gamma^{2}}{2} \int_{0}^{T} \operatorname{Var}_{t}\left(d \mathbb{E}_{t}\left[\hat{R}_{T}\right]\right)}$ in the description of the equivalence in Section 4.1. To determine $\epsilon$, we define $Z_{t}:=\mathbb{E}_{t}\left[\hat{R}_{T}\right]=f\left(t, X_{t}\right)+R_{t}$, which is a martingale, hence

$$
\begin{aligned}
d Z_{t} & =\frac{\partial f}{\partial x} \nu\left(t, X_{t}\right) d B_{t}^{X}+\hat{\pi}_{t} \sigma_{t} d B_{t} \\
& =\left(\frac{\partial f}{\partial x} \nu\left(t, X_{t}\right)+\rho \hat{\pi}_{t} \sigma_{t}\right) d B_{t}^{X}+\sqrt{1-\rho^{2}} \hat{\pi}_{t} \sigma_{t} d B_{t}^{\perp} \\
d\langle Z\rangle_{t} & =\left[\left(\frac{\partial f}{\partial x} \nu\left(t, X_{t}\right)+\rho \hat{\pi}_{t} \sigma_{t}\right)^{2}+\left(1-\rho^{2}\right)\left(\hat{\pi}_{t} \sigma_{t}\right)^{2}\right] d t \\
& =\left[\left|\sigma_{t} \hat{\pi}_{t}\right|^{2}-2 v_{t} \sigma_{t} \hat{\pi}_{t} / \gamma+\left(\frac{\partial f}{\partial X} \nu\left(t, X_{t}\right)\right)^{2}\right] d t .
\end{aligned}
$$


Hence

$$
\begin{aligned}
\varepsilon=e^{-\frac{\gamma^{2}}{2}\langle Z\rangle_{T}} & =e^{\gamma \int_{0}^{T}\left(-\frac{\gamma}{2}\left|\sigma_{t}^{\prime} \hat{\pi}_{t}\right|^{2}+v_{t} \sigma_{t} \hat{\pi}_{t}\right) d t} e^{\left.-\int_{0}^{T}\left(\gamma \frac{\partial f}{\partial X} \nu\left(X_{t}\right)\right)^{2} / 2\right] d t} \\
& =e^{\gamma \int_{0}^{T}\left[r_{t}+\hat{\pi} \sigma_{t}\left(\theta_{t}+v_{t}\right)-\frac{1+\gamma}{2}\left|\sigma_{t} \hat{\pi}_{t}\right|^{2}\right] d t} e^{-\int_{0}^{T}\left(\gamma \nu\left(X_{t}\right) \frac{\partial f\left(t, X_{t}\right)}{\partial X}\right)^{2} / 2 d t-\gamma \int_{0}^{T} a_{t} d t} \\
& =A_{T} e^{-\gamma Y_{0}+\gamma \int_{0}^{T} r_{t} d t} \frac{d \mathbb{Q}^{v}}{d \mathbb{P}}
\end{aligned}
$$

For any portfolio $\pi$., denote $W$. as its wealth process starting from initial $W_{0}=w$, then

$$
\begin{aligned}
\frac{1}{-\gamma} W_{T}^{-\gamma} & =\frac{w^{-\gamma}}{-\gamma} e^{-\gamma \int_{0}^{T}\left(r+\pi_{t} \sigma_{t}\left(\theta_{t}+v_{t}\right)-\left|\sigma_{t} \pi_{t}\right|^{2} / 2\right) d t-\int_{0}^{T} \gamma \pi_{t} \sigma_{t} d B_{t}^{v}} \\
& =\frac{\left(w e^{r T}\right)^{-\gamma}}{-\gamma} e^{-\gamma \int_{0}^{T}\left(\pi_{t} \sigma_{t}\left(\theta_{t}+v_{t}\right)-(1+\gamma)\left|\sigma_{t} \pi_{t}\right|^{2} / 2\right) d t} e^{-\int_{0}^{T}\left|\gamma \sigma_{t} \pi_{t}\right|^{2} / 2 d t-\int_{0}^{T} \gamma \pi_{t} \sigma_{t} d B_{t}^{v}} \\
& \leq \frac{\left(w e^{r T}\right)^{-\gamma}}{-\gamma} \frac{1}{A_{T}} e^{-\int_{0}^{T}\left|\gamma \sigma_{t} \pi_{t}\right|^{2} / 2 d t-\int_{0}^{T} \gamma \pi_{t} \sigma_{t} d B_{t}^{v}}
\end{aligned}
$$

and the equality hold if $\pi=\hat{\pi}$. Hence

$$
\begin{aligned}
\mathbb{E}\left[\varepsilon \frac{W_{T}^{-\gamma}}{-\gamma}\right] & =e^{-\gamma Y_{0}} \mathbb{E}_{\mathbb{Q}^{v}}\left[A_{T} e^{\gamma \int_{0}^{T} r_{t} d t} \frac{W_{T}^{-\gamma}}{-\gamma}\right] \\
& \leq e^{-\gamma Y_{0}} \frac{w^{-\gamma}}{-\gamma} \mathbb{E}_{\mathbb{Q}^{v}} e^{-\int_{0}^{T}\left|\gamma \sigma_{t} \pi_{t}\right|^{2} / 2 d t-\int_{0}^{T} \gamma \pi_{t} \sigma_{t} d B_{t}^{v}} \leq e^{-\gamma Y_{0}} \frac{w^{-\gamma}}{-\gamma} .
\end{aligned}
$$

When $\pi=\hat{\pi}$, the equality in the first inequality holds; Under some regularity condition, the equality in the second inequality holds, hence $\hat{\pi}$ is an optimal portfolio.

\section{C.7. Convergence of the Hedge Ratio to That in Basak and Chabakauri [4] as $\gamma \rightarrow \infty$ for the Stochastic Return Model}

Denote $\mathbb{E}[\hat{\pi}(t, \bar{X})]=F(\gamma) \frac{\bar{X}}{\sigma(1+\gamma)}$. Then $\frac{\mathbb{E}\left[\hat{H}\left(t, X_{t}\right)\right]}{\mathbb{E}[\hat{\pi}(t, \bar{X})]}=\frac{F(\gamma)-1}{F(\gamma)}$. Sending $\gamma \rightarrow \infty$, we get $b=\lambda+\rho \nu$ and $q=|\lambda+\rho \nu|$. A direct calculation gives

$$
\begin{aligned}
\lim _{\gamma \rightarrow \infty} F(\gamma) & =1-2 \rho \nu \frac{|\lambda+\rho \nu|\left(e^{2|\lambda+\rho \nu|(T-t)}-1\right)+\lambda\left(e^{|\lambda+\rho \nu|(T-t)}-1\right)^{2}}{|\lambda+\rho \nu|\left[((\lambda+\rho \nu)+|\lambda+\rho \nu|)\left(e^{2|\lambda+\rho \nu|(T-t)}-1\right)+2|\lambda+\rho \nu|\right]} \\
& =1-\rho \nu\left\{\frac{1-e^{-2(\lambda+\rho \nu)(T-t)}}{\lambda+\rho \nu}+\lambda \frac{\left(1-e^{-(\lambda+\rho \nu)(T-t)}\right)^{2}}{(\lambda+\rho \nu)^{2}}\right\},
\end{aligned}
$$

which is desired.

\section{C.8. The Proof for the Gaussian Time-Varying Mean-Return Model with Two Stocks}

To be consistent with the notations in Proposition 4, we define $B_{1 t}=\hat{B}_{1 t}, B_{2 t}=\frac{\hat{B}_{2 t}-\rho_{S} \hat{B}_{1 t}}{\sqrt{1-\rho_{S}^{2}}}$. It follows

$$
\sigma=\left(\begin{array}{cc}
\sigma_{1} & 0 \\
\rho_{S} \sigma_{2} & \sqrt{1-\rho_{S}^{2}} \sigma_{2}
\end{array}\right), \quad \rho=\left(\rho_{1}^{S X}, \frac{\rho_{2}^{S X}-\rho_{S} \rho_{1}^{S X}}{\sqrt{1-\rho_{S}^{2}}}\right)^{\top}
$$


A direct calculation gives (40).

The PDE that $f(t, x)$ satisfies has the same structure as that in Proposition 2. Using the same approach as in the proof of Proposition 2 we can show the monotonicity of $\frac{\partial f}{\partial x}$ with respect to the investment horizon. 